\title{
Measurement of River Surface Currents with Coherent Microwave Systems
}

\author{
William J. Plant, William C.Keller, Kenneth Hayes \\ Applied Physics Laboratory \\ University of Washington \\ Seattle, Washington 98105
}

January 19, 2005

\begin{abstract}
River surface currents have been measured using coherent microwave systems from a bridge, a cableway, several riverbanks, a helicopter, and an airplane. In most cases, the microwave measurements have been compared with conventional measurements of near-surface currents and found to be accurate to within about $10 \mathrm{~cm} / \mathrm{s}$. In all cases, the basis for the microwave measurement of surface current is the Doppler shift induced in the signal backscattered from the rough water surface. In this paper, we outline the principles of the measurements and the various implementations that have been used to make microwave measurements of surface currents. CW microwave systems have been used from a bridge to make long-term measurements of surface currents; these are compared with current-meter measurements and with time series of stage. A compact CW system has been developed and used on a cableway to measure surface currents at various distances across a river; these measurements have been compared with acoustic ones. Pulsed Doppler radars have been used to measure river surface currents from a riverbank, a helicopter, and an airplane. In the first two cases comparisons with both current-meter and acoustic measurements have been made. We suggest that the CW system would be preferable to the pulsed Doppler radar to make such measurements from helicopters in the future. Finally, we consider the implications of our experiments for the measurement of surface currents from aircraft or satellites using interferometric synthetic aperture radars (INSARs). We find that a combination along-track, crosstrack INSAR is necessary but that significant limitations are inherent in the technique.
\end{abstract}

\section{Introduction}

Routine measurements of river surface heights (stage), velocity, and volume discharge have been conducted by the US Geological Survey (USGS) in essentially the same manner for the last hundred years. The technique consists of constructing gaging stations along rivers to monitor the stage of the river and periodically calibrating these data to yield the discharge. The calibrations consist of insitu measurements of river velocity and depth, which, when measured at intervals across the river, completely determine its discharge (volume of water per unit time). Velocities and depths are measured by USGS personnel either by using a bridge, wading the river, using a small boat, or operating from a cableway across the river. Construction of cableways capable of carrying people and installing gaging stations are significant expenses, as is the manpower-intensive periodic measurement of the discharge. Recently, innovations have been introduced in which velocities and depths are measured by acoustic means or by tracking drifters using the Global Positioning System (GPS). 
All of these techniques require that instruments be placed in the water. This subjects the instruments to fouling, which adds considerable maintenance expense to the other expenses of the method. Furthermore, handling instruments in the water can be hazardous in high flow conditions. Debris floating down the river can damage instruments and endanger the people making the measurements. The standard means of measuring currents and depths from cableways, for instance, is for the operator to suspend a current meter from a cablecar over the water using a cable with a heavy weight on the end. The purpose of the weight is to locate the bottom and maintain the cable in a vertical position against the force of the flow. Lowering such a weight can be a dangerous operation during high flows.

For these reasons a means of measuring river discharge remotely would be highly desirable and, in fact, a promising means of doing so has recently been reported (Costa, et.al., 2000). This technique consists of using coherent microwave radars to measure surface currents and lower-frequency, ground-penetrating radars (GPRs) to measure the depth. The Japanese have also used radar to measure surface currents (Yamagucyi and Niizato, 1994) and the USGS and the Japanese have previously used GPR to measure river depth (Spicer, et al, 1997; Okamoto, Y., 1999). Very recently ultra-high frequency (UHF) signals have been used to measure surface currents (Teague, et al., 2003). The purpose of the present paper is to describe coherent microwave techniques for surface current measurement, to demonstrate their accuracy when used from bridges, cableways, riverbanks and helicopters, and to discuss the possibility of measuring river surface currents from space using microwave interferometric synthetic aperture radar (INSAR).

\section{Principles of Surface Current Measurement using Coherent Microwaves}

The physical phenomenon used to measure surface currents with microwaves is the Doppler shift, whereby moving objects change the frequency of a signal scattered from them. In order to detect the complete spectrum of Doppler shifts, the transmitted signal must be coherent, that is, it must have a time series with no phase discontinuities. River surface currents were measured in our experiments using the Doppler shift induced in microwave signals backscattered from river surfaces due to advection of the scatterers by the current. When microwaves are incident on rough water surfaces at incidence angles that are not too large or small, they are scattered back to the antenna by short surface waves a few centimeters in length. This process is known as composite surface scattering in which Bragg-resonant scattering from short surface waves occurs independently from small facets on the water surface that are tilted and advected by larger scale motions (Wright, 1968; Bass et al., 1968; Plant, 1990, Plant and Keller, 1990). Lengths of those short waves that cause backscatter are well characterized by the Bragg resonance condition

$$
\lambda_{b}=\frac{\lambda}{2 \sin \theta}
$$

where $\lambda_{b}$ is the wavelength of the resonant water wave (the Bragg wave), $\lambda$ is the microwave length, and $\theta$ is the incidence angle. For an X-band radar, $\lambda=3 \mathrm{~cm}$ so $\lambda_{b}$ is about 1.5 to 2 $\mathrm{cm}$. 
Due to diffraction effects, typical microwave antennas have beamwidths on the order of a few degrees. Thus areas on the water surface illuminated by such antennas are decimeters to meters in size in each dimension. This illumination pattern ensures that only Bragg waves traveling toward or away from the antenna in the plane of incidence are effective scatterers. Short waves travelling in other directions are averaged out of the scattering process by the illuminated area. The scattering process can be described in many conditions as a two-dimensional Fourier transformation of the surface wave spectrum in which only those particular components with wavenumbers along the horizontal antenna look direction that satisfy the Bragg resonance condition contribute to the scattering (Plant, 1990). In still water, surface waves propagate at a well-known characteristic phase speed, $c=|\vec{c}|$, which has its minimum value of $23 \mathrm{~cm} / \mathrm{s}$ near $\lambda_{b}=1.7 \mathrm{~cm}$. When a current of horizontal velocity $\vec{v}$ is present in the water, these waves are advected by the current so that their speed relative to the riverbank is $\vec{v}+\vec{c}$. Thus the Doppler shifts produced by the two possible scatterers are:

$$
f_{d}=-\frac{2 V_{l}}{\lambda}=\frac{-2(\vec{v}+\vec{c}) \cdot \overrightarrow{a_{r}}}{\lambda}=\frac{v_{h} \pm c}{\lambda_{b}}
$$

where $V_{l}$ is the line-of-sight velocity, that is, the component of scatterer velocity along the antenna look direction. Also, $\overrightarrow{a_{r}}$ is a unit vector in the direction of transmission and $v_{h}$ is the component of $\vec{v}$ along the horizontal antenna look direction. Removing the frequency of the transmitted signal from the received signal and taking the spectrum yields a Doppler spectrum of the received signal. The two well-defined Doppler shifts given by Eq.(2) are displayed as sharp peaks, commonly called "Bragg lines", in the Doppler spectrum and are said to come from "advancing" (positive Doppler shifts) or "receding" (negative Doppler shifts) Bragg waves. Figure 1a shows simulated Doppler spectra displaying these two possible Bragg lines for no current (solid lines) and with a current present (dashed lines).

The situation becomes more complex when variations in the mean current are taken into account. These variations may be produced either by turbulence of the flow or by surface waves longer than the Bragg wavelength. The effect of these current variations is to advect the Bragg waves at a variety of speeds, thus broadening the spectrum of Doppler shifts around the two frequencies given in Eq.(2). Long surface waves also tilt the Bragg waves slightly, varying the local incidence angle and producing additional broadening. In cases where this broadening is not too severe, two broadened Bragg lines can still be detected in the spectrum of the backscattered signal (See Figure 1b and Plant and Keller, 1990). Severe broadening causes the Bragg lines to overlap to such an extent that the existence of advancing and receding waves cannot be detected (Figure 1c).

A further complication is the source of the Bragg waves. Such short waves may be naturally produced directly by the wind, indirectly by longer waves, by the turbulence of the water, or by rainfall (Plant, 1997; Plant, 2003; Contreras, 2004). When produced by wind, the short waves have their maximum intensity in the direction of the wind and decrease in intensity symmetrically about the wind direction as the azimuth angle to the wind increases, becoming very weak, but not nonexistent, when travelling against the wind. Much the same intensity variations are present when the Bragg waves are produced by longer waves. Rain falling on a water surface produces a variety of splash products but the ones primarily affecting microwave backscatter in most cases are the ring waves produced by the drops 

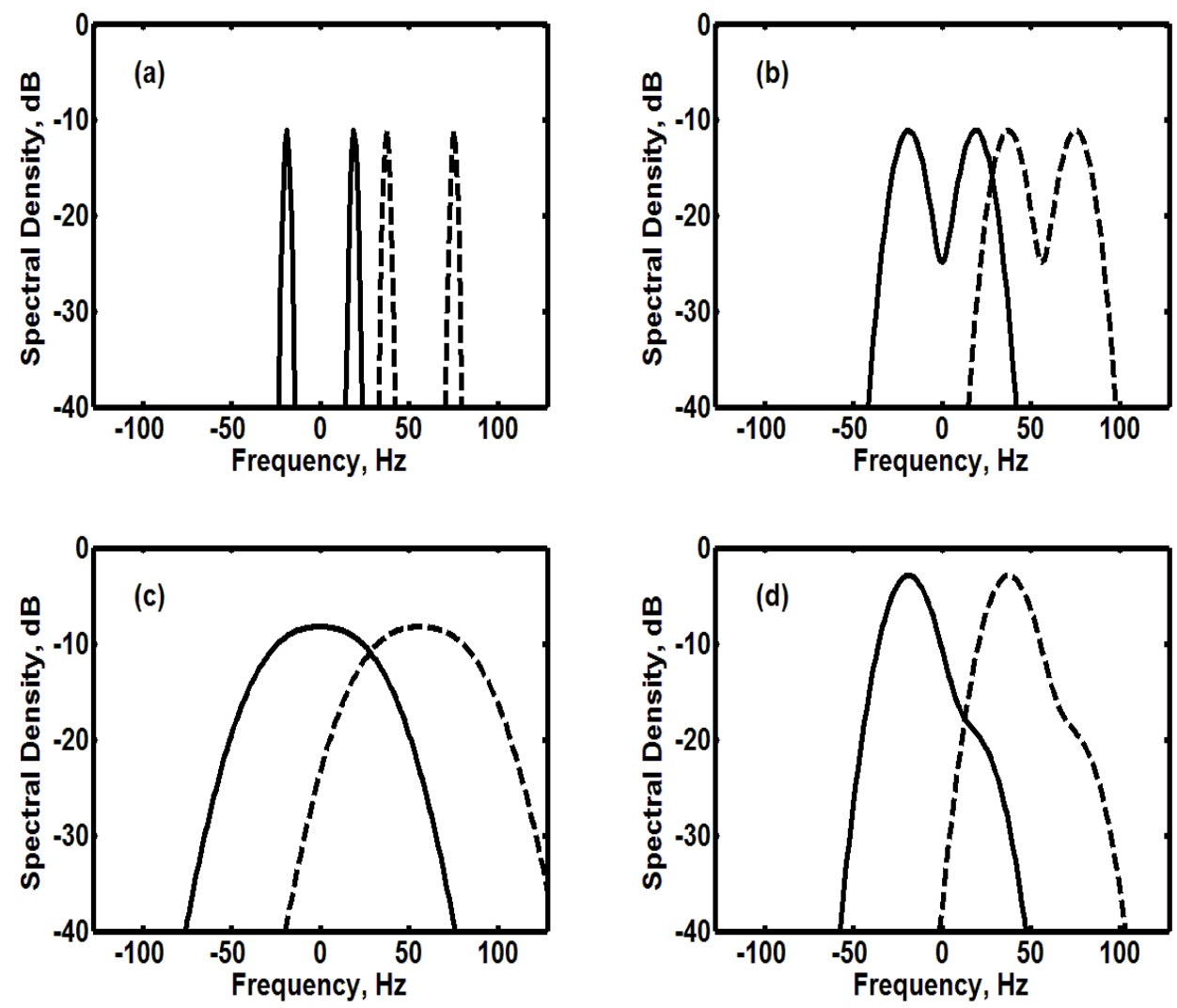

Figure 1: Simulations of various types of Doppler spectra observed in microwave backscatter. a) Bragg waves of equal amplitude present on the surface, as during rainfall. b) Equalamplitude Bragg wave moderately advected by larger-scale motions. c) Equal amplitude Bragg waves severely advected by larger-scale motions. d) Bragg waves of unequal amplitude moderately advected by larger-scale motions, as for wind-generated waves looking nearly along the wind direction. 
(Braun et al., 2002; Contreras et al., 2004). These are symmetric so that no azimuthal intensity variation is present in short waves produced by rainfall. When the short waves are produced by turbulence, the azimuth angle dependence of their intensity has not been well established to date.

Because composite surface theory shows that the intensity of a Bragg line is proportional to the intensity of the surface wave producing it, the azimuth angle variations in Bragg wave intensity result in differing strengths (spectral densities) of the two Bragg lines. This must be taken into account when deriving a mean surface current from a measured Doppler spectrum. Figure 1d illustrates the case when the Bragg lines are broadened and have different spectral densities. An extreme situation of the type illustrated in Figure 1d is when the weaker Bragg line disappears into the noise baseline of the spectrum. This may be the case when the waves are generated by the wind and the antenna is directed with or against the wind. The difference between this case and that of two equal-intensity but very broadened Bragg lines (Figure 1c) may be difficult to discern.

For all possible types of Doppler spectra, Eq.(2) shows that the frequency midway between those of the two Bragg lines must be determined in order to measure the surface velocity of the river. The variety of possible spectra makes this difficult in general unless care is taken in choosing this frequency. Errors in choosing the proper frequency can result in errors in the measured current up to $\pm c$, which is at least $\pm 23 \mathrm{~cm} / \mathrm{s}$. We have developed algorithms to choose this frequency correctly given the complete Doppler spectrum and use the same algorithms in the most systems to be described in the following sections.

Even if this midpoint frequency is chosen correctly, another possible source of error in these measurements occurs due to the drift current caused at the surface by the wind. The effective depth at which the velocity is measured in a microwave measurement is approximately $0.044 \lambda_{b}$ (Plant and Wright, 1980). For $\lambda_{b}=1.7 \mathrm{~cm}$, this depth is about $0.75 \mathrm{~mm}$. At this depth, the wind drift layer has not completely decayed. The magnitude of the wind drift at the surface is well known from measurements, however, to be about $2 \%$ of the wind speed measured $10 \mathrm{~m}$ above the surface and in the wind direction. For a $10 \mathrm{~m} / \mathrm{s}$ wind speed, this is $20 \mathrm{~cm} / \mathrm{s}$. Assuming a logarithmic decay to the effective depth of the microwave measurement following Plant and Wright (1980), the wind drift at the effective measurement depth is about $11 \mathrm{~cm} / \mathrm{s}$. This amount of error is incurred in the measurement of surface velocity, of course, only if the wind blows exactly along the direction in which the antenna is pointing. For other directions, the error will be less. As the results in the following sections show, this small error due to the wind drift is not a problem for the measurements in general. If one desired, the wind vector could be measured along with the microwave measurements and a correction could be made for the wind drift. This has not been done in the results reported below.

Under low wind and flow conditions, sufficient surface roughness may not be present to produce backscatter above system noise levels. Thus under such conditions, the techniques presented in this paper may not yield a measurement. This will vary from one system to another depending on the noise level. In any case, such conditions are not difficult to measure by traditional mean. The microwave techniques presented here are expected to work well at moderate to high flows and may prove especially valuable during floods. 


\section{Summary of Measurements}

In the following sections, we report the results of a series of studies on measuring river surface currents with both pulsed and continuous-wave (CW) coherent microwave systems from bridges, cableways, riverbanks, helicopters, airplanes, and satellites. For reference, Table 1 summarizes the various methods examined, the instruments used, their frequencies and spatial resolutions, and whether they are measurements at a single small area (point), a horizontal profile, or a map of the river. The final column of the table assesses the viability of the various techniques based on the results of these studies. In the table, Riverscat is a continuous wave microwave system, RiverRad is a pulsed Doppler radar, CORAR is an airborne real aperture coherent radar, and ATI SAR is an along-track interferometric synthetic aperture radar.

\begin{tabular}{|c|c|c|c|c|c|}
\hline Location & Instrument & Frequency & Approx. Res. & Type & Viability \\
\hline \hline Bridge & CW Systems & $24 \mathrm{GHz}$ & $2 \mathrm{~m}$ by $3 \mathrm{~m}$ & Point & Very Good \\
\hline Cableway & Riverscat & $24 \mathrm{GHz}$ & $2 \mathrm{~m}$ by $3 \mathrm{~m}$ & Profile & Very Good \\
\hline Riverbank & RiverRad & $10 \mathrm{GHz}$ & $4 \mathrm{~m}$ to $30 \mathrm{~m}$ & Profile & Excellent \\
\hline Helicopter & CORAR & $9.36 \mathrm{GHz}$ & $2 \mathrm{~m}$ by $10 \mathrm{~m}$ & Profile & Excellent \\
\hline Airplane & CORAR & $9.36 \mathrm{GHz}$ & $10 \mathrm{~m}$ by $10 \mathrm{~m}$ & Map & Questionable \\
\hline Air/Space & ATI SAR & Various & $3 \mathrm{~m}$ by $3 \mathrm{~m}$ & Map & Questionable \\
\hline
\end{tabular}

Table 1: Summary of Techniques Studied. For riverbank measurements, only range resolution is shown because azimutal resolution varies widely. Point indicates a measurement at a single point; profile represents a horizontal profile across the river; and map indicates that velocities can be mapped over the river.

\section{Measurements from the Ground}

\subsection{Continuous-Wave Measurements from a Bridge}

The simplest way to implement a microwave technique for measuring river surface currents is to use a homodyne transiever that can be purchased very cheaply. We attached eight such transievers operating at $24 \mathrm{GHz}$ to a like number of vertically polarized, one-foot diameter, parabolic antennas, amplified the received signals, and fed them to a data acquisition system. This system digitized each of the eight analog signals at a $1 \mathrm{kHz}$ rate, computed a Doppler spectrum every half second, and stored the results in files for subsequent processing. Spectra were analyzed by fitting the noise level to an inverse frequency function, dividing the received signal by this noise level, and applying an algorithm to determine the frequency, $f_{c}$ midway between the Bragg lines. The surface velocity, $v_{h}$, in the direction of the horizontal look of the antenna was then determined from the equation

$$
v_{h}=\frac{\lambda f_{c}}{2 \sin \theta}
$$




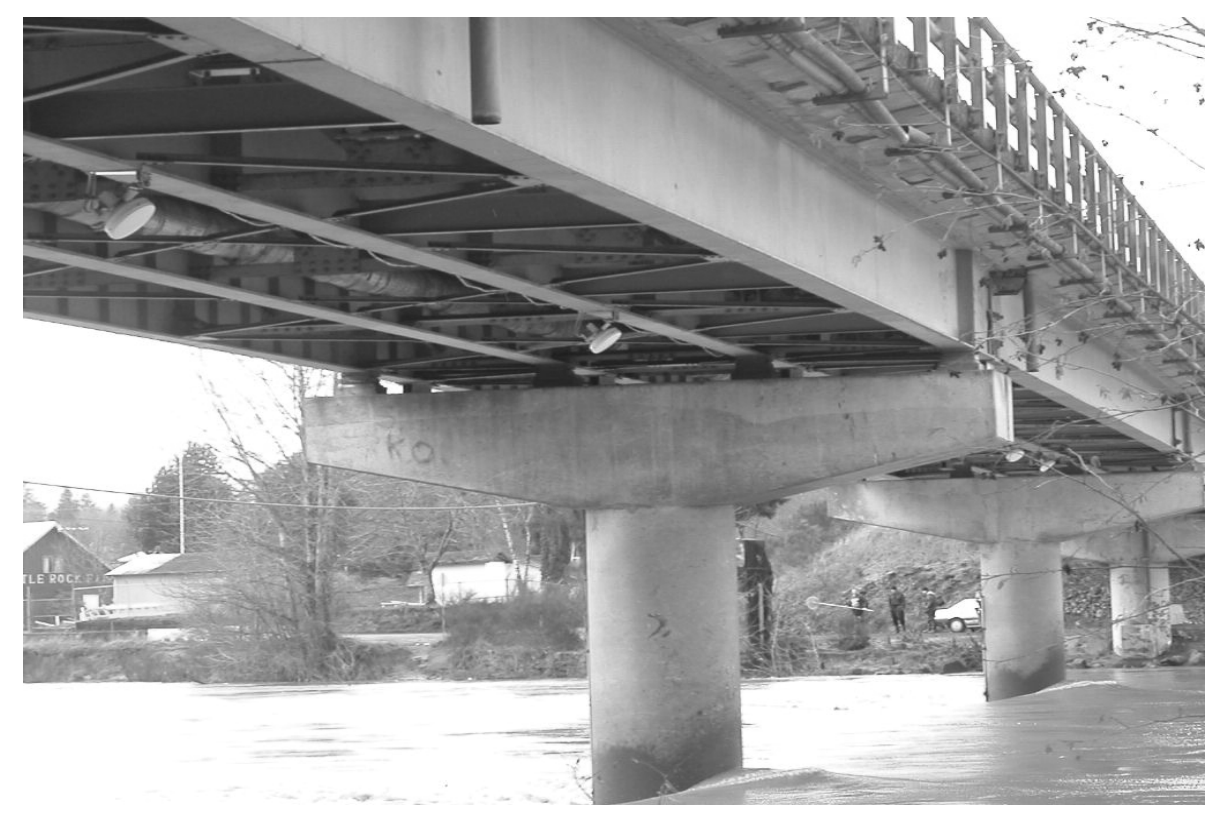

Figure 2: Four of the eight continuous wave microwave systems mounted on the Cowlitz River bridge at Castle Rock, WA.

Since these are homodyne systems, Doppler spectra are wrapped, that is, are only given at positive frequencies irrespective of the direction of travel of the scatterers. Thus the sign of $v_{h}$ cannot be determined from these measurements; one must know the flow direction.

These eight microwave velocity sensors were mounted on the Cowlitz River bridge at Castle Rock, WA, the site of USGS gage 14243000, in September, 2002. They were spaced at intervals of approximately $10 \mathrm{~m}$. Each antenna was mounted under the bridge at a height of about $10 \mathrm{~m}$, depending on river stage, and at an incidence angle of $45^{\circ}$. Six of the eight antennas were pointed directly upstream while the other two were directed at $30^{\circ}$ to the upstream direction. Each antenna transmits approximately $5 \mathrm{~mW}$ of power in a beam whose one-way, half-power width is approximately $3^{\circ}$. Figure 2 shows four of these eight sensors on the bridge. Figures 3 and 4 give examples of Doppler spectra collected by these sensors during rainfall (Figure 3) and during clear conditions (Figure 4). The two Bragg peaks are conspicuous during rainfall but not so obvious under clear conditions. The figures show the locations of $f_{c}$ picked by our algorithms and give the measured surface velocities at the top of each panel. Antenna locations are also given at the top of each panel in terms of distance from the left side of the bridge. Comparisons of the surface velocities determined from these eight microwave sensors with those measured by the USGS using an AA current meter measuring as close to the surface as possible (about $18 \mathrm{~cm}$ below the mean surface) are shown in Figure 5. The left side of the bridge is defined as the side on the left when facing downstream. Additional comparisons are given in Plant et al., 2004a.

Velocities from these microwave sensors have now been collected every thirty minutes for several months. During low flow conditions, generally at surface velocities below about $1 \mathrm{~m} / \mathrm{s}$, these sensors do not yield a measurement unless the wind speed is above about 3 $\mathrm{m} / \mathrm{s}$ or rain is falling. Thus gaps in the data record can and do occur. Even when signals are obtained, cases exist when only a single Bragg line can be observed so the systems yield 

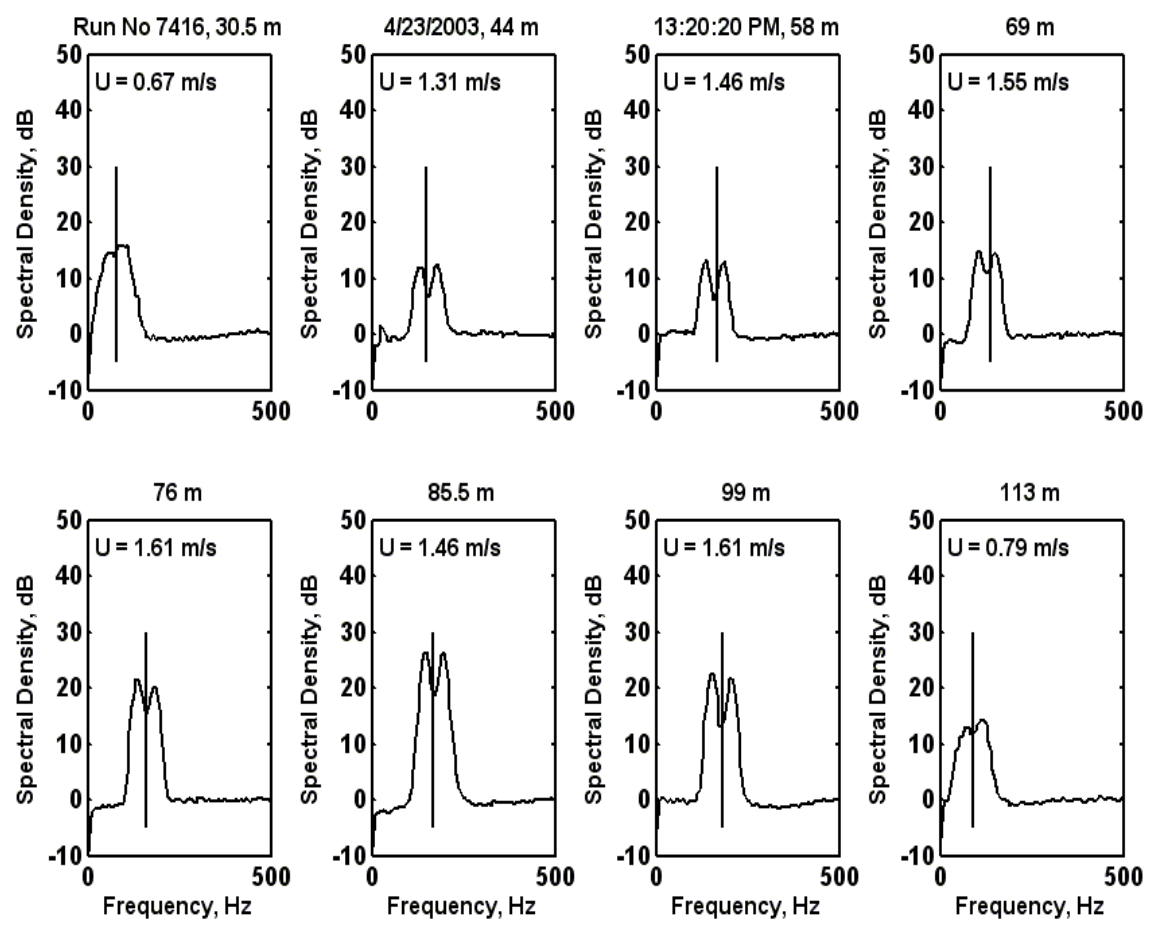

Figure 3: Doppler spectra collected by the eight microwave surface velocity sensors mounted on the Cowlitz River bridge during rain. Measured velocities are shown in each panel. Vertical lines are the chosen center frequency, $f_{c}$. Distances above each panel are the distance from the left side of the bridge to the sensor. 

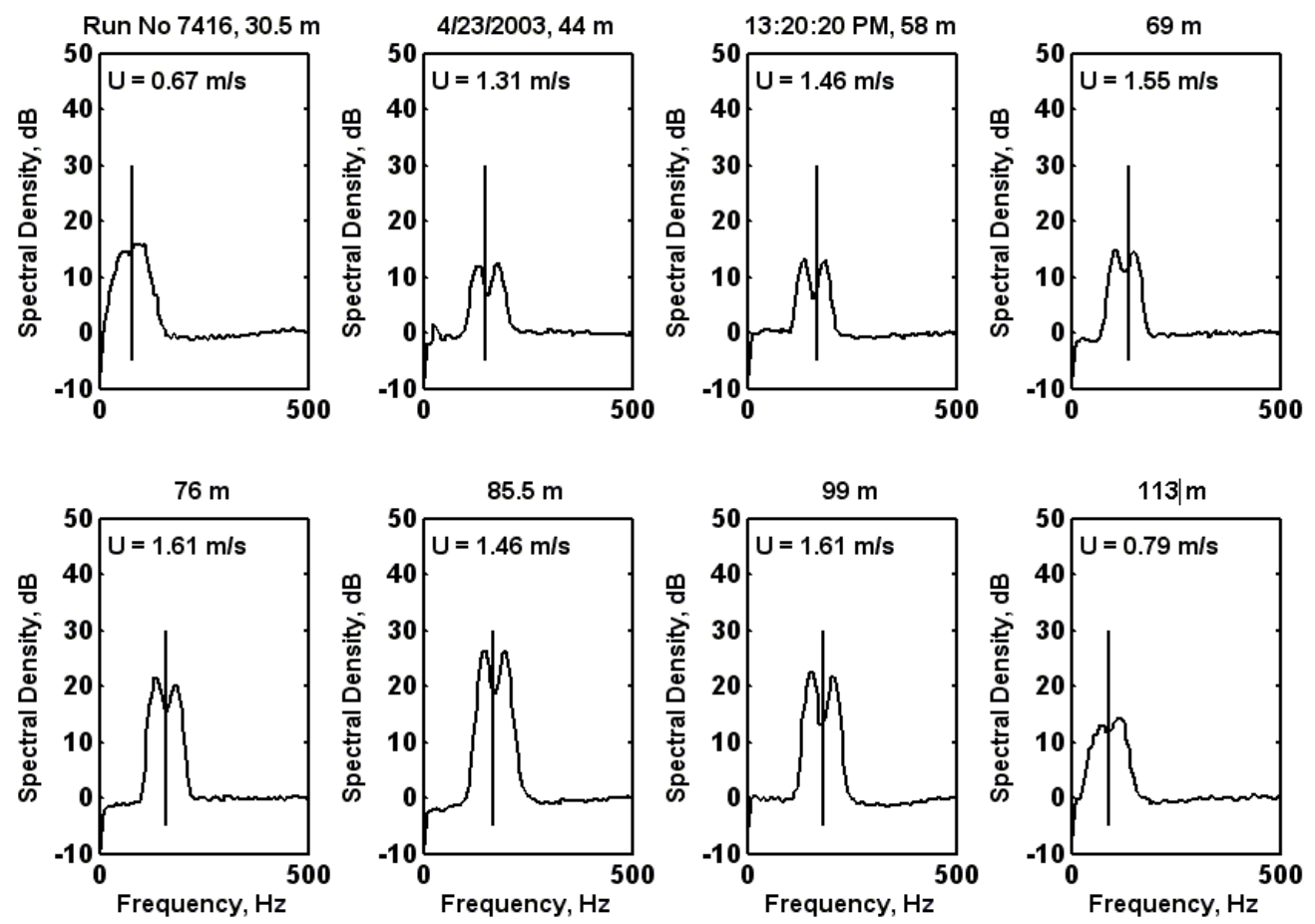

Figure 4: Same as Figure 3 but under clear conditions.
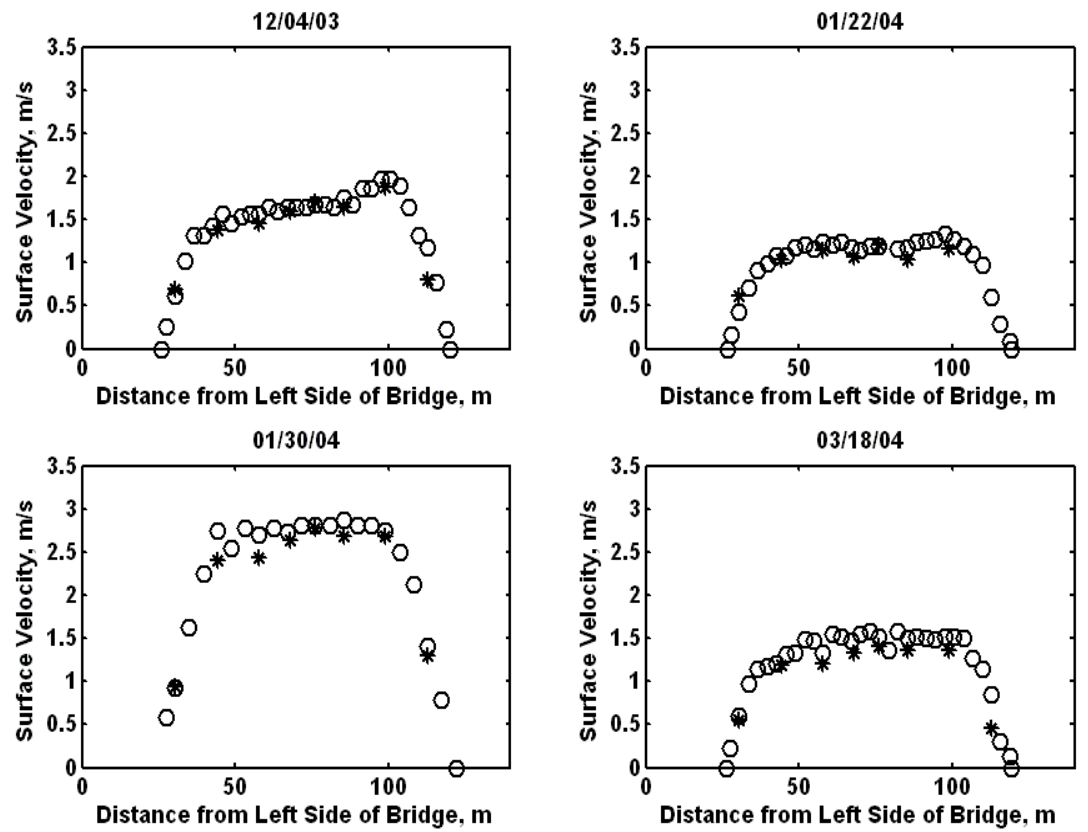

Figure 5: Comparison of surface velocities measured by the bridge-mounted continuous-wave microwave sensors (Asterisks) with those measured by an AA current meter (circles). 

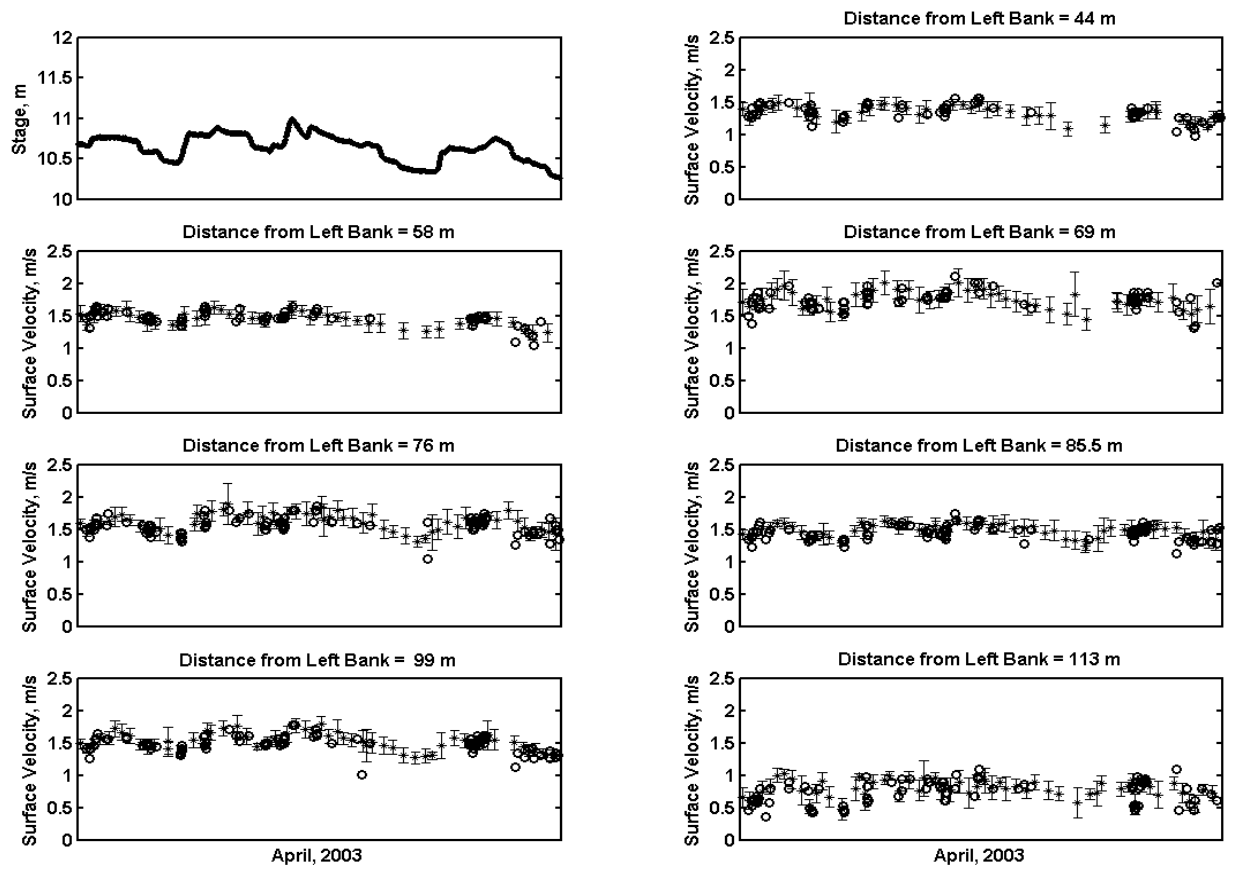

Figure 6: Surface velocities derived from seven of the eight microwave velocity sensors on the Cowlitz River bridge at Castle Rock, WA during the month of April, 2003. The upper left plot shows the time series of stage measured by the USGS during the month. In the other plots, open circles indicate data collected during rain and show individual measurements. Asterisks indicate data taken under clear conditions. Four hours of data, about 8 measurements have been averaged to yield the asterisks; error bars are standard deviations.

two possible values for $f_{c}$ at $\pm f_{b}$ on either side of the observed peak, where $f_{b}=c / \lambda_{b}$ is the frequency of the Bragg-resonant surface wave (See Figure 4 at $44 \mathrm{~m}$ ). In order to eliminate one of these two possible values from the time series, the nearest unambiguous velocity (from situations with rain or a favorable wind direction) was determined and used to choose one of the two possible ambiguous values. Following this procedure, four hours of velocity values, usually eight values, were averaged together to yield a time series of surface velocities.

One such time series is shown in Figure 6 for the month of April, 2003. In the velocity plots, open circles indicate data collected during rain. They represent unambiguously determined surface velocities and have not been averaged over four hours. Asterisks indicate averaged data with error bars that are plus and minus one standard deviations over a fourhour period. Clearly very small variations in the stage of the river are reflected in changes in the measured surface velocities. Plant et al., 2004a give more details of the correlation between surface velocity and stage and the causes of this correlation. 


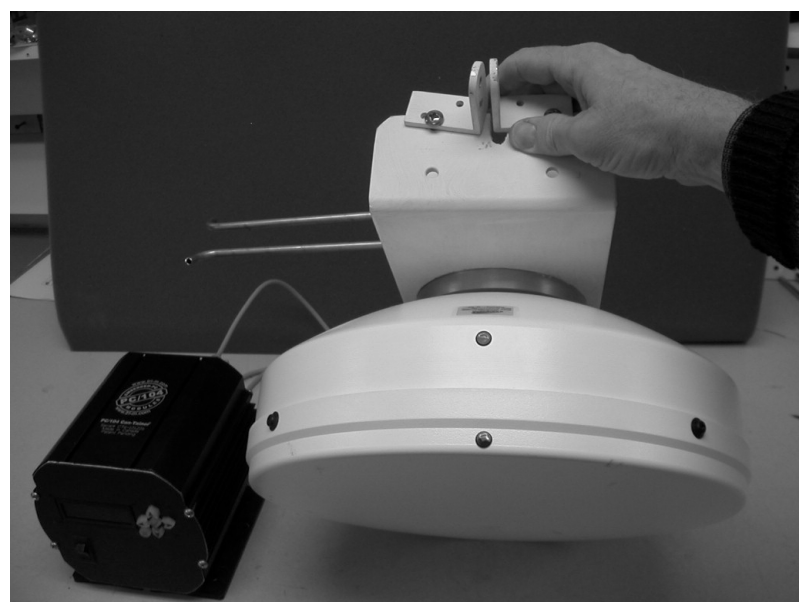

Figure 7: The RiverScat microwave surface velocity sensor.

\subsection{Continuous-Wave Measurements from a Cableway}

Following these measurements from the bridge, we miniaturized these $24 \mathrm{GHz}$ continuouswave microwave sensors to make it possible to use them from a cableway. A data collection and analysis system was developed around a PC-104 486 computer module using the Linux operating system. This module also contained a compact flash disc and an A/D card. The data acquisition and data analysis functions of the bridge-mounted systems were combined as part of the computing module so that surface velocities could be displayed on a liquid crystal screen in real time. These velocities and the intermediate Doppler spectra were stored on the compact flash for subsequent use. The computer module was designed so that up to four antenna units could be attached. The resulting computer module/antenna system was called RiverScat and is shown in Figure 7. The two tubes protruding from the side of the antenna are pitot tubes that allow the component of the wind along the horizontal antenna-look direction to be determined. This is necessary in the algorithm that finds $f_{c}$ when trying to separate the cases shown in Figures 1c and 1d.

In order to make complete discharge measurements from a light cableway, RiverScat with a single antenna was mounted on top of the antenna of a ground penetrating radar owned by the USGS. This arrangement is shown in Figure 8 as configured on the Cowlitz River near Castle Rock, WA. The package was sufficiently light that it could be towed across the river by an electric motor on the bank. No personnel were required to be on the cableway as in the standard USGS operating procedure.

The RiverScat units have now been operated from the light cableway over the Cowlitz River near Castle Rock, WA many times since October, 2003. Figure 9 gives an example of the type of data obtained from the system. Two to four traverses across the river, taking a total of less than 30 minutes, have been averaged together to produce the displayed plots. Also shown on the plots are near-surface velocities measured by a floating acoustic system called BoogieDopp. 


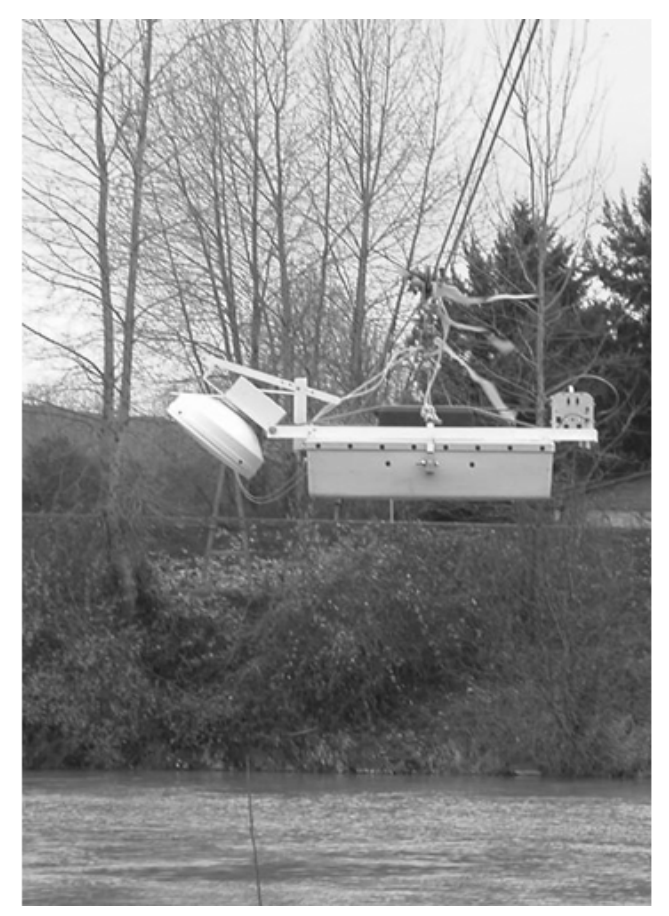

Figure 8: RiverScat mounted on the antenna of the USGS ground-penetrating radar. Both are being towed across the Cowlitz River near Castle Rock, WA on a light cableway operated from the bank.
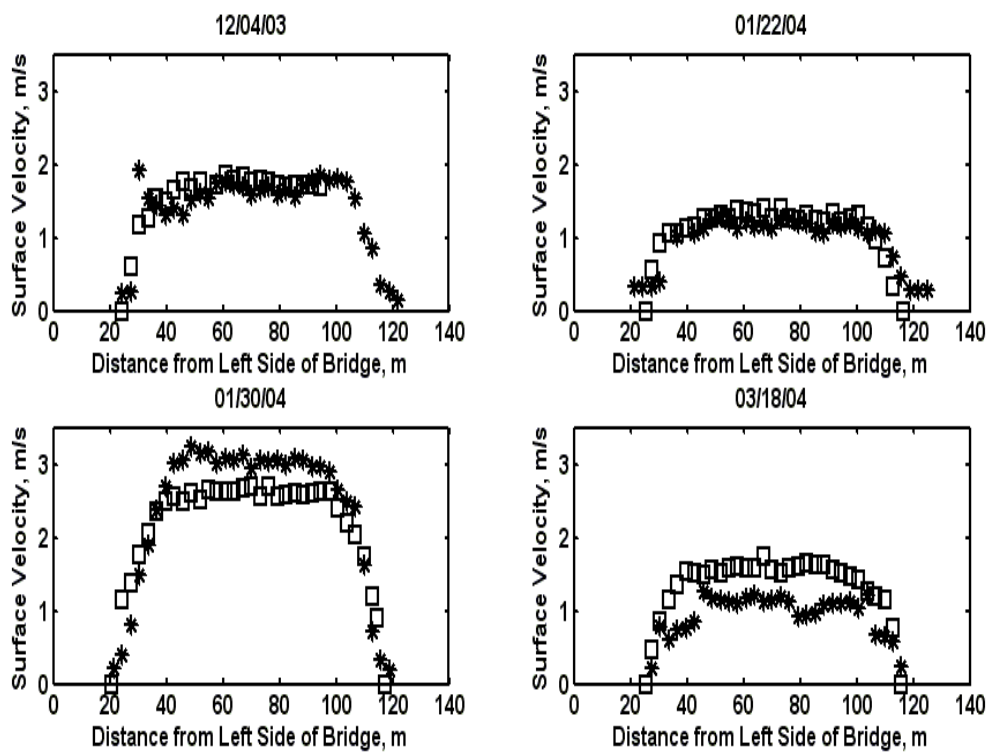

Figure 9: Comparison of surface velocities obtained by RiverScat on the light cableway over the Cowlitz River near Castle Rock, WA (asterisks) with measurements using an acoustic system called BoogieDopp (squares). 


\subsection{Pulsed Doppler Radar operated from a Riverbank}

During the past few years, we have made measurements of river surface currents from several riverbanks using two different, but very similar, pulsed Doppler radars. The radars were coherent, X-band radars designed and constructed at APL/UW.

The first, which we call CORAR for COherent Real Aperture Radar, operated at 9.36 GHz. It was built during a previous project dealing with ocean measurements that was funded by the Office of Naval Research (Plant et al., 2004b) and adapted to river measurements. The primary adaptations involved the antennas where we replaced the slottedwaveguide antennas that operated at both $\mathrm{HH}$ and VV polarization (electric field horizontal $(\mathrm{HH})$ or vertical (VV) on both transmit and receive) with one or two parabolic antennas 60 $\mathrm{cm}$ in diameter operating only at VV polarization. The radar transmitted 50 nsec pulses with a $40 \mathrm{kHz}$ pulse repetition frequency. Peak transmitted power was approximately 100 watts, which had proven sufficient over the ocean to detect backscatter under all but the lowest wind speeds. CORAR is a hetrodyne system that uses an intermediate frequency of 160 $\mathrm{MHz}$. Output of the radar consisted of inphase (I) and quadrature (Q) signals proportional to the received field that had been beat down to baseband.

These signals were each sampled at $20 \mathrm{MHz}$ by an A/D board and processed by a data acquisition system, also designed and constructed at APL/UW. The data acquisition system first summed 32 pulses coherently to increase the signal-to-noise ratio (SNR) and then computed the mean power, $I^{2}+Q^{2}$, received from each resolution cell, which was $7.5 \mathrm{~m}$ long in the range direction. Pulse compression was not used. The system further computed Doppler spectra at each of 100 range bins. Because of the coherent averaging, each range bin could be sampled at a maximum rate of $1250 \mathrm{~Hz}$. The system was designed, however, to allow averaging of a variable number of pulses while maintaining a constant sample rate. This was accomplished by inserting a delay between the averaged pulses so that the sample rate always stayed at $1000 \mathrm{~Hz}$. Thus the Nyquist frequency of CORAR was $500 \mathrm{~Hz}$, and the maximum line-of-sight velocity that could be accomodated by the antenna without producing an aliased spectrum was $8 \mathrm{~m} / \mathrm{s}$.

From the Doppler spectra, Doppler offsets and bandwidths, computed as the first and second moments of the spectrum, were obtained by the data acquisition system. Received power, Doppler offsets, and Doppler bandwidths were then stored on hard disk for each of the 100 range bins. In addition, a complete spectrum from a single, selected bin was stored along with the other information. This complete procedure - sampling, processing, and storing - took a total of approximately $360 \mathrm{msec}$ and was considered to be one scan; alternate scans came from alternate antennas if two were used. Figure 10 shows CORAR being operated from the bank of the Skagit River near Mt. Vernon, WA in 1999. Due to the narrow beamwidth of the parabolic antenna used in this experiment, it was necessary to collect data at three different, small grazing angles in order to cover the whole river. Results of these measurements have been reported by Costa et al., 2000.

The second radar used in these studies was a modification of CORAR, which we called RiverRad. The transmitted frequency was shifted slightly to $10 \mathrm{GHz}$. The transmitted pulse length was made variable in factors of two from $3.75 \mathrm{~m}$ to $30 \mathrm{~m}$. The transmitted power was reduced to 10 watts and the maximum number of pulses that could be coherently averaged was increased to 60 . The pulse repetition frequency was $39.0625 \mathrm{kHz}$ and the sample rate was 


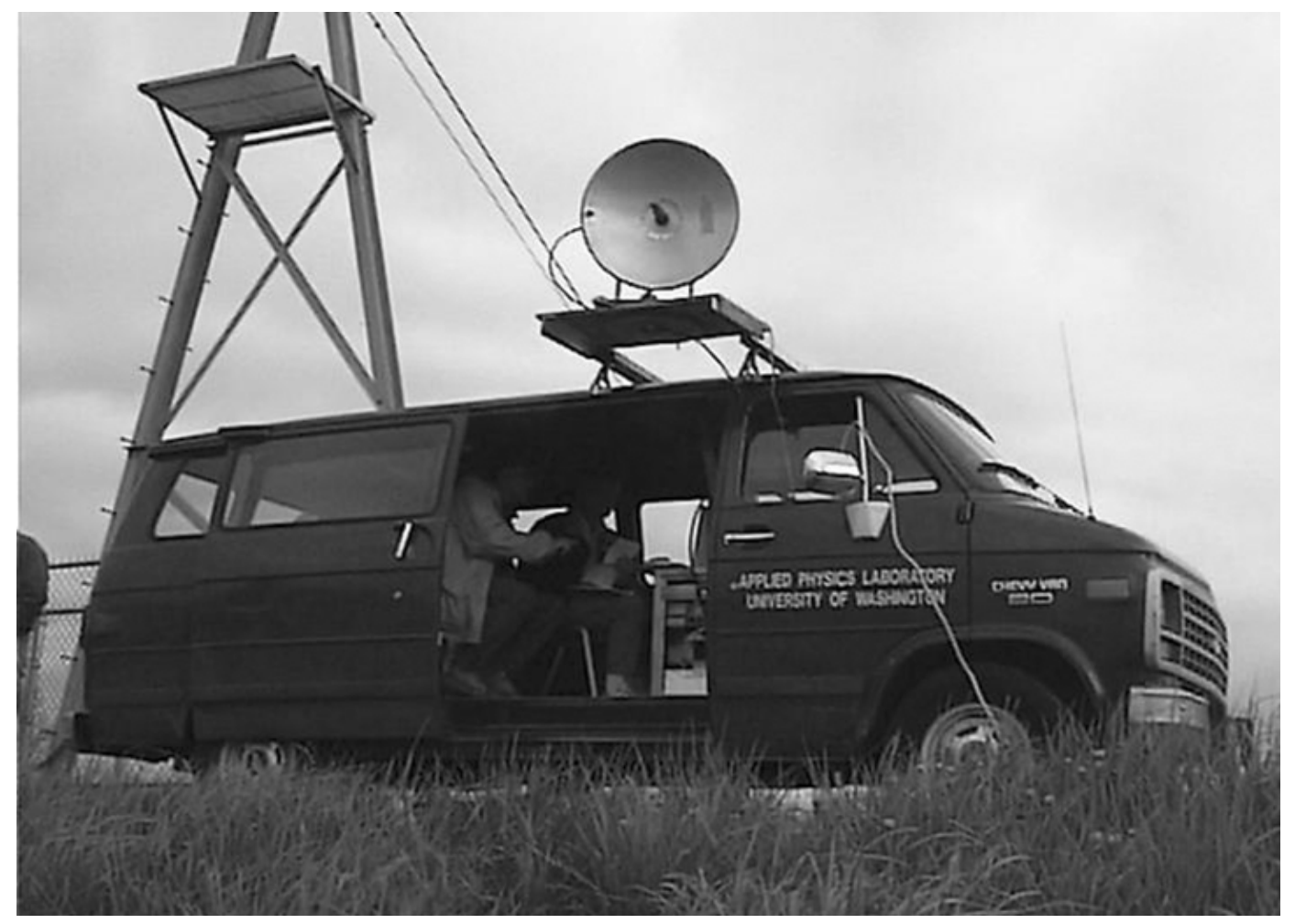

Figure 10: CORAR operating from the bank of the Skagit River near Mt. Vernon, WA in 1999.

allowed to vary with the number of pulses averaged coherently. Thus the sample rate when returns from $\mathrm{N}$ pulses were averaged coherently was $39062.5 / \mathrm{N} \mathrm{Hz}$. Doppler spectra were stored at every range bin, thus increasing the time between scans. In order to aleviate the necessity for varying the grazing angle of the antenna, the vertical beamwidth of the $60-\mathrm{cm}$ parabolic antenna used with RiverRad was broadened by modifying the antenna. Switching between two different antennas, which was automatic in CORAR unless manually disabled, became an operator choice in RiverRad. When the transmitted signal was automatically switched between two antennas, one pointing slightly upstream and one pointing slightly downstream, the radar could collect data without operator intervention. The radar was also repackaged to be easier to operate in remote locations. Figure 11 shows RiverRad in operation with two antennas on the bank of the San Joaquin River near Vernalis, CA in 2002; the insert shows the complete radar, with only a single antenna, in the laboratory.

In order to obtain the largest component of the current toward the radar antenna when operating from the riverbank, we initially tried to look up or down river at angles to the perpendicular that were as large as possible. While this procedure works, it is hard to implement in practice since it is often difficult to find locations where trees permit open lines of sight nearly along the river. Furthermore, currents can vary significantly along the river, making such measurements difficult to compare with the standard procedures where measurements are made along the perpendicular. Eddies in rivers can create local areas of significant cross river flow which is also measured by the radar but not by current meters. To avoid as many of these complications as possible, we developed a technique similar to 


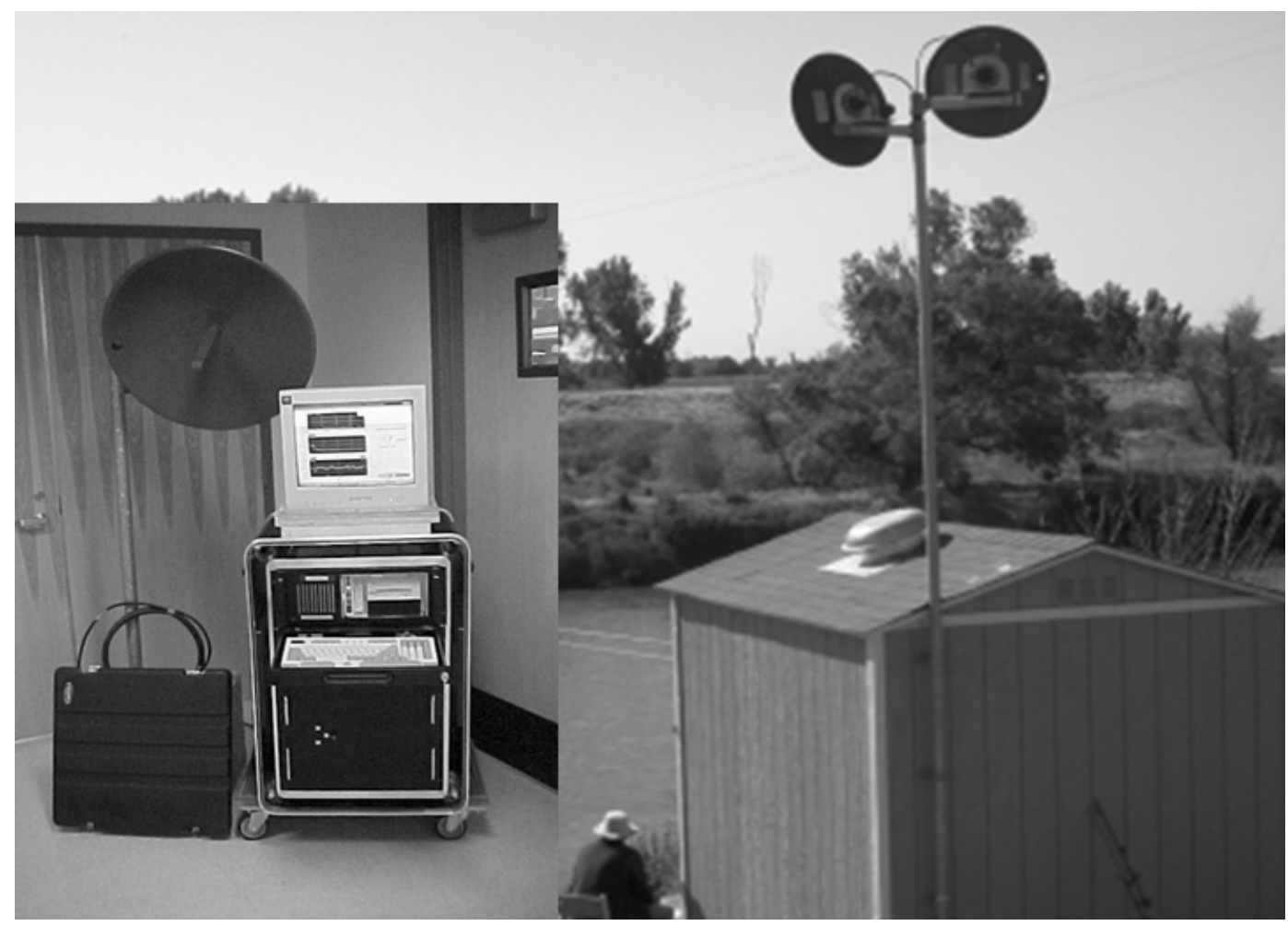

Figure 11: RiverRad operating from the bank of the San Joaquin River near Vernalis, CA in 2002.

that used in laser Doppler velocimetry. We directed the antenna of the bank-mounted radar first upstream at a small angle to the cross-stream direction then downstream at a small angle, or used two antennas for the same purpose.

Before going farther, it is convenient to establish some conventions to be used in the remainder of the paper. Let the surface current vector be written in terms of its components along and across the river, $u$ and $v$, respectively. As shown in Figure 12, the positive direction of $v$ is taken to be toward the left bank. Then we measure the pointing direction, $\phi$, of an antenna clockwise from the positive $v$ direction as shown in the figure. Thus, Eq.(2) written in this coordinate system is

$$
f_{d}=-\frac{V_{l}}{\lambda_{b} \sin \theta}=-\frac{(u \sin \phi+v \cos \phi) \pm c}{\lambda_{b}}
$$

where velocities toward the antenna produce positive Doppler shifts and those away from the antenna produce negative shifts.

Using these conventions, then, and assuming that the current is the same at the two slightly different measurement locations, the central Doppler shifts (ideally, the midpoint between Bragg lines) obtained with the antennas pointing slightly upstream, $f_{d u}$, and downstream, $f_{d d}$, may be written

$$
f_{d u}=-\frac{u \sin \phi_{u}+v \cos \phi_{u}}{\lambda_{b}}
$$




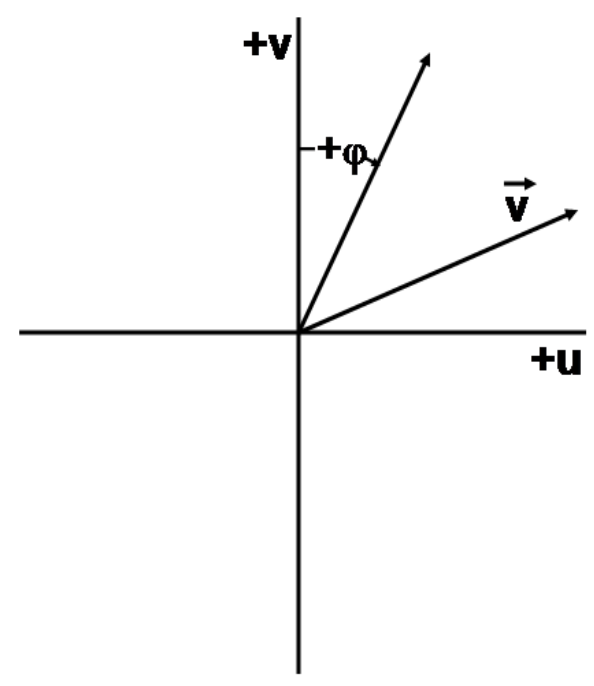

Figure 12: Coordinate system and angle conventions used in this paper. The instantaneous current velocity is indicated by $\vec{v}$, current speed in the direction of mean flow is $u$, and that across the river is $v$. The angle $\phi$ measures the angle between $+v$ and the horizontal look-direction of the antenna.

and

$$
f_{d d}=-\frac{u \sin \phi_{d}+v \cos \phi_{d}}{\lambda_{b}}
$$

Multiplying appropriately by sines and cosines and subtracting (6) from (5), it is easy to find $\mathrm{u}$ and $\mathrm{v}$ :

$$
\begin{aligned}
& u=\frac{\lambda_{b}\left(f_{d d} \cos \phi_{u}-f_{d u} \cos \phi_{d}\right)}{\sin \left(\phi_{u}-\phi_{d}\right)} \\
& v=\frac{\lambda_{b}\left(f_{d d} \sin \phi_{u}-f_{d u} \sin \phi_{d}\right)}{\sin \left(\phi_{u}-\phi_{d}\right)}
\end{aligned}
$$

For runs using CORAR, $f_{d u}$ and $f_{d d}$ where taken to be the first moments of the Doppler spectrum, $\mathrm{S}(\mathrm{f})$,

$$
f_{d}=\frac{\int f S(f) d f}{\int S(f) d f}
$$

so they will include effects of $\vec{c}$ if the intensities of the advancing and receding Bragg waves are not equal. For runs using RiverRad, more accurate algorithms were used to choose the midpoint frequency between the Bragg lines independent of their intensity ratios.

Since 1998, we have made measurements with pulsed Doppler radars operating from the banks of the Green, Skagit, and Cowlitz Rivers in western Washington state and the 
San Joaquin River in California. The measurements on the Green and Skagit Rivers used CORAR; those on the Skagit River have been reported elsewhere (Costa et al., 2000). Measurements on the Cowlitz and San Joaquin Rivers were made using RiverRad. Comparisons of the latter measurements with in situ measurements of surface velocity made nearly simultaneously by the USGS are shown in Figure 13.

\section{Measurements from the Air}

\subsection{Measurements from a Helicopter}

In 2000 and 2001, we conducted a series of experiments with CORAR mounted on a Bell 206B Jet Ranger helicopter. CORAR was modified slightly from the configuration employed on the riverbank to use separate antennas on transmit and receive so that signals from shorter range could be received without interference from ringing of the transmitted pulse. A set of transmit/receive antennas, vertically polarized slotted waveguide antennas $45 \mathrm{~cm}$ long, were mounted on each side of the helicopter. Alternate scans came from alternate sides of the helicopter. The helicopter was flown as perpendicular to the river flow as possible. The outfitted helicopter is shown in Figure 14 in flight over the Cowlitz River. The helicopter carried a ground-penetrating radar owned by the USGS as well as CORAR so that complete discharge measurements could be obtained. Results have been reported by Melcher, et al., 2002. The helicopter was equiped with a differential GPS receiver for accurately determining its location. This GPS was not able to give helicopter attitude so an Attitude and Heading Reference System (AHRS) was mounted in the tail. The AHRS gave heading from a compass and pitch and roll from gyros. The downwash from the helicopter caused a very strong signal to be returned from the surface to the microwave antennas. Data were collected at altitudes ranging from 1.5 to $12.2 \mathrm{~m}$. We checked how far away from the helicopter the radar could receive a return and found that it had decreased into the noise at a range of $43 \mathrm{~m}$ when we flew at $1.5 \mathrm{~m}$ and at $70 \mathrm{~m}$ at an altitude of $12.2 \mathrm{~m}$. However, long before this point, the low signal-to-noise ratio had caused the Doppler shift to be too low (Plant et al., 1998). This occurred at all altitudes by the seventh range bin, or about $50 \mathrm{~m}$ range. We chose to use our fourth range bin for the measurements. Due to a slight delay in the one channel, this yielded the range of $24.4 \mathrm{~m}$ on one side of the helicopter and $30 \mathrm{~m}$ on the other. Results reported here were obtained at an altitude of $7.6 \mathrm{~m}$ so our incidence angles to the interrogated spots on the river surface were $72^{\circ}$ and $75^{\circ}$.

Velocities for each channel were computed by first locating the footprint on the water surface then using Eqs. (2) and (4). The yaw of the helicopter varied across the river and was measured by the compass in the AHRS. Because of this yaw and non-perpendicular flight across the river, the antenna footprints on either side of the helicopter were located at different distances across the river. Thus one side had to be shifted in order to match the location on the other side. Because the helicopter downdraft is roughly symmetrical in

azimuth and always directed outward from the helicopter, the direction of $\vec{c}$ in Eq.(2) was known to be radially outward, and therefore, always away from the microwave antennas. 

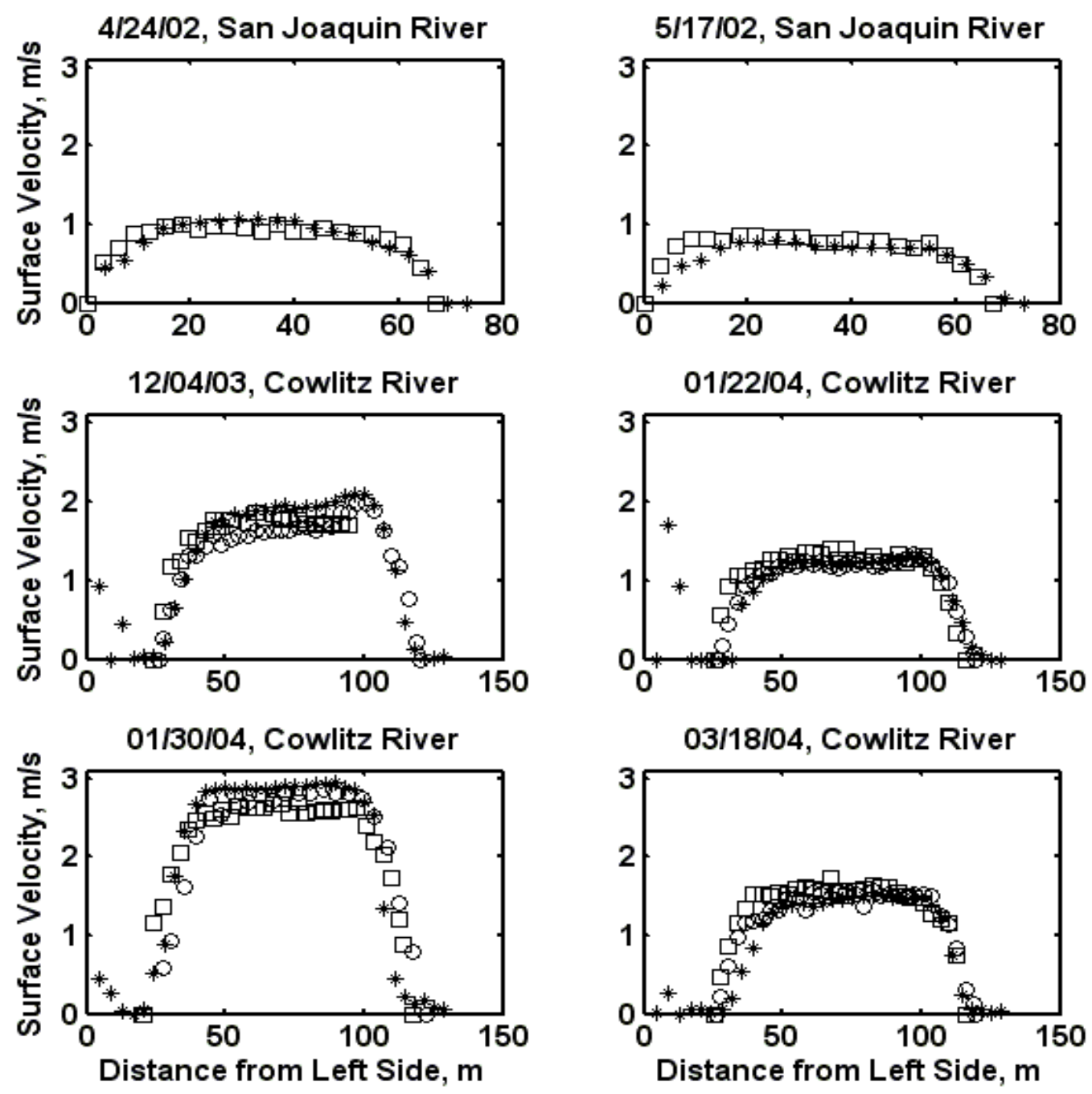

Figure 13: Comparison of RiverRad surface velocity measurements with in situ values. On the San Joaquin River, RiverRad measurements (asterisks) were collocated with the BoogieDopp measurements (squares). On the Cowlitz River, RiverRad (asterisks) was located midway between the AA current meter on the bridge (circles) and the acoustic "BoogieDopp" system on the cableway (squares). 


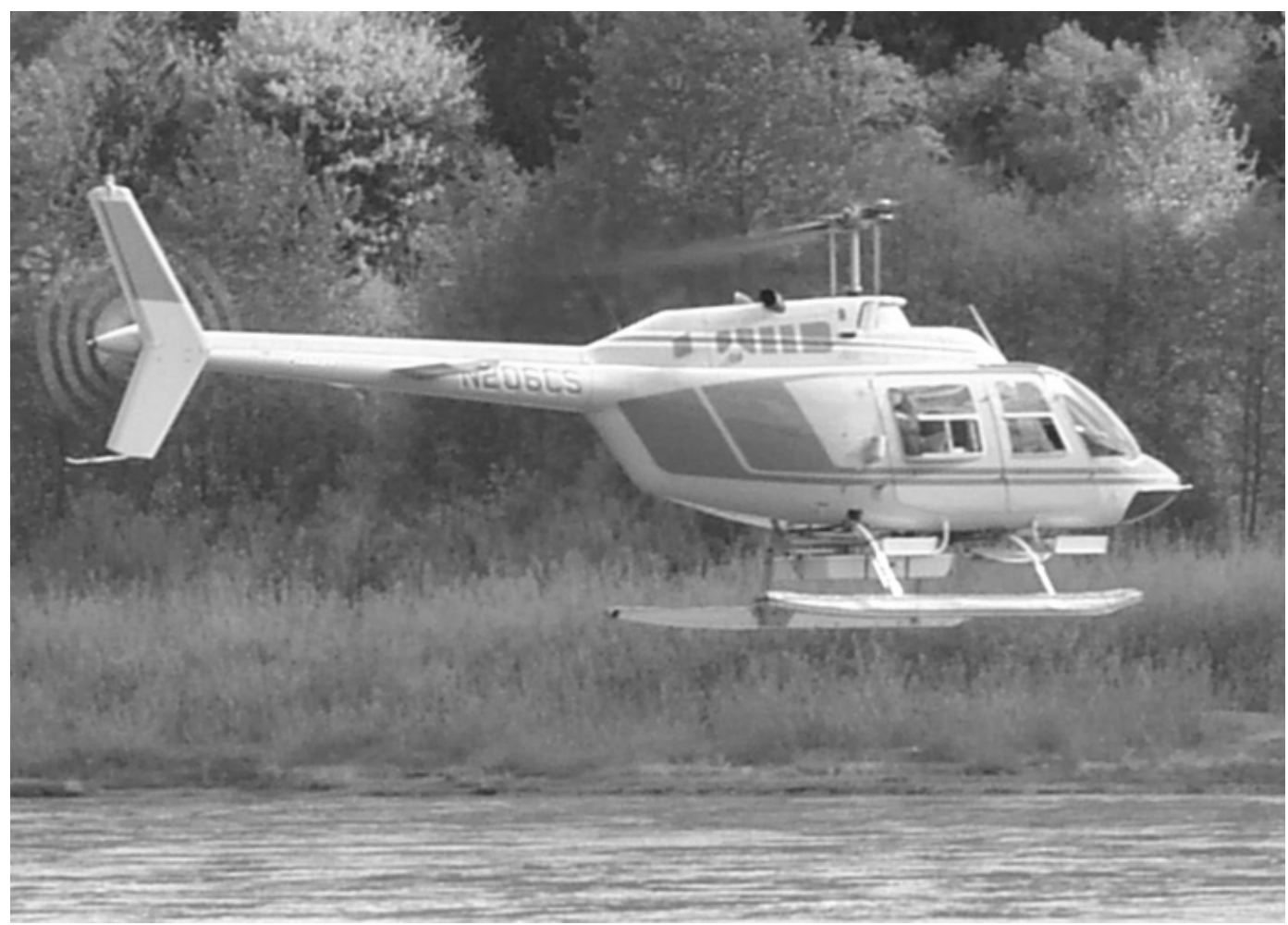

Figure 14: The Jet Ranger helicopter in flight over the Cowlitz River near Castle Rock, WA. The large white object under the cabin is the USGS ground-penetrating radar. The two small, white antennas on the sides are the X-band microwave waveguide antennas. An identical pair was located on the other side of the helicopter

Thus Eq.(4) yields

$$
f_{d}= \pm \frac{\left(u \cos \left(\gamma+\phi_{y}\right)-v \sin \left(\gamma+\phi_{y}\right)+V \sin \phi_{y}\right)}{\lambda_{b}}-\frac{c}{\lambda_{b}}
$$

where $\mathrm{V}$ is the helicopter speed, $\gamma$ is the angle between the ground track from the GPS and the $+v$ direction, and $\phi_{y}$ is the yaw of the helicopter, that is, the heading from the AHRS minus the track given by the GPS. The top sign refers to measurements from the antennas on the left side of the helicopter, the lower sign to the right side. Subtracting the Doppler shift obtained looking right, $f_{d r}$, from that obtained looking left, $f_{d l}$, neglecting the small cross stream term, and solving for $u$ yields

$$
u=\frac{\lambda_{b}\left(f_{d l}-f_{d r}\right)-2 V \sin \phi_{y}}{2 \cos \left(\gamma+\phi_{y}\right)} .
$$

Note that the phase speed, c, of the Bragg scatterers does not appear in this result since it affects $f_{d l}$ and $f_{d r}$ in the same manner. On the other hand, this equation clearly shows the importance of accurately knowing both the yaw of the helicopter and its flight direction relative to the $+v$ direction. The impact of yaw can be reduced by reducing the helicopter speed. Thus for surface velocity measurements, it is desirable to traverse the river as slowly 
as possible. Helicopter speeds in our experiments were approximately $1 \mathrm{~m} / \mathrm{s}$. We found that the headings measured by the AHRS were too large by about $8^{\circ}$. Figure 15 compares surface velocities across the Cowlitz River obtained from in situ ADCP and AA current meters with those obtained from the microwave measurements for various incremental reductions of the yaw given by the AHRS. The $8^{\circ}$ increment clearly yields the best match to the in situ data.

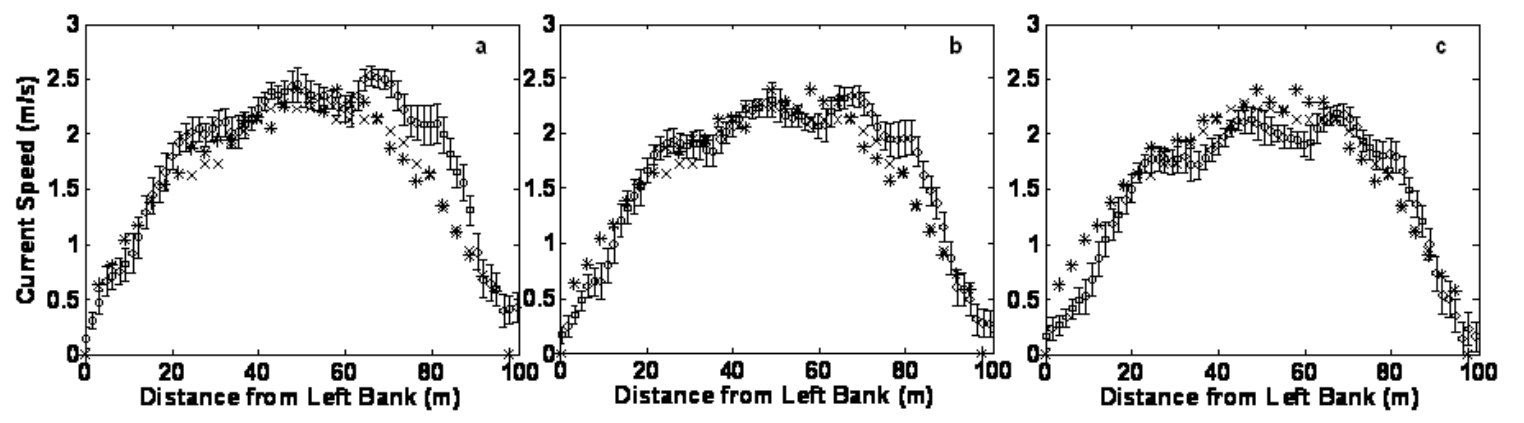

Figure 15: Surface currents obtained from CORAR mounted on the helicopter (circles with error bars) compared with those measured by ADCP (x) and AA current meters (+). The different panels show surface currents derived from the helicopter measurements subtracting various corrections from the measured yaw: a) $4^{\circ}$, b) $8^{\circ}$, and c) $12^{\circ}$

While these measurements using CORAR clearly demonstrated the ability of the radar to measure surface velocities from a helicopter it is undoubtedly not the optimum microwave instrument to use for this purpose. CORAR is bulky - the electronics occupied one whole passenger seat - and difficult to install in the helicopter. RiverScat would be much easier to install and operate on a helicopter and its performance on the cableway indicates that it could make the measurements. We have designed RiverScat with inputs for GPS and AHRS signals so, in principle, it could be mounted in the helicopter and flown unattended across the river to obtain the measurements. We intend to try this during our next helicopter measurements. 


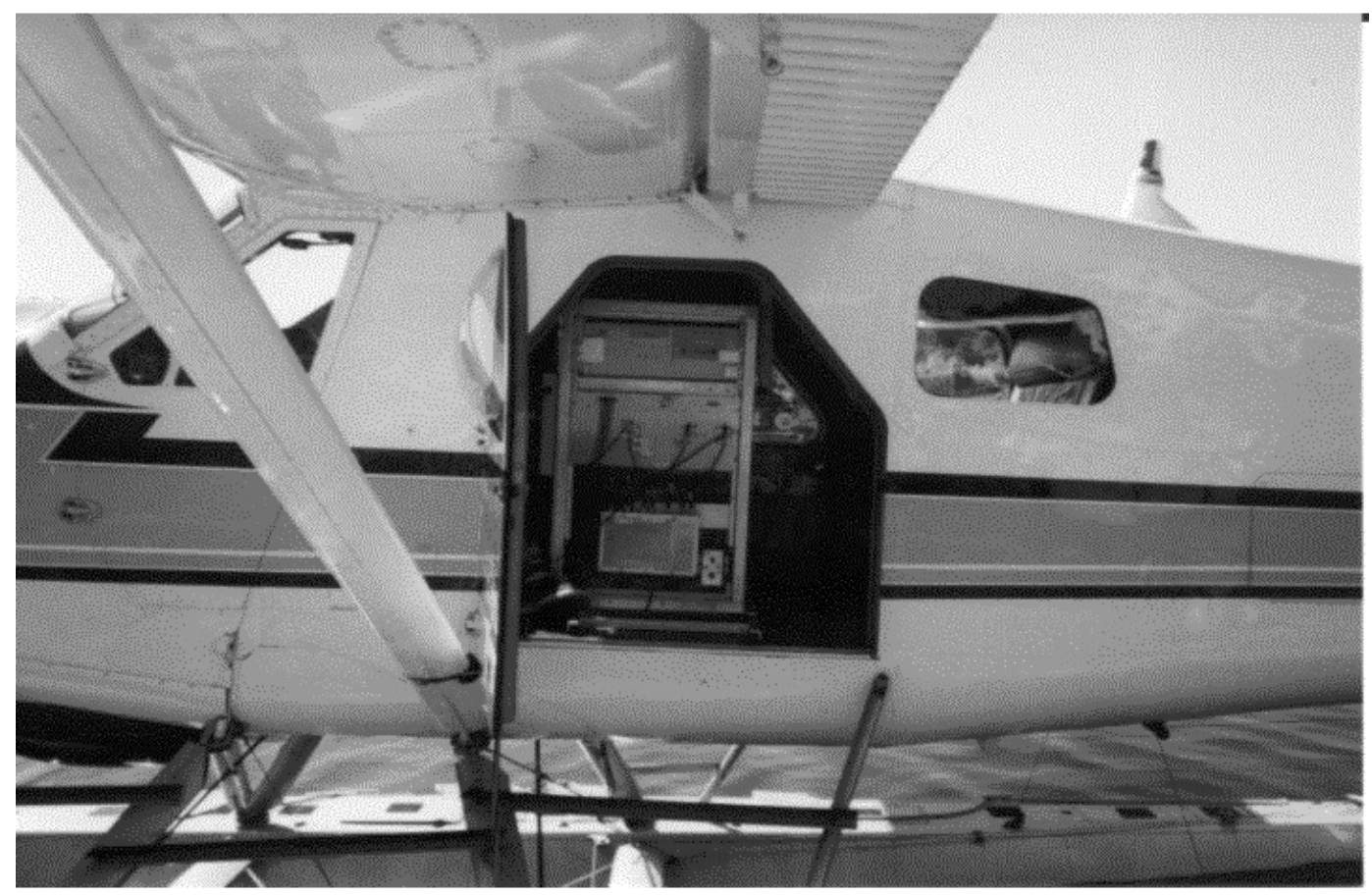

Figure 16: The DeHaviland Beaver with the electronics rack of CORAR mounted inside.

\subsection{Pulsed Doppler Radar on a Light Aircraft}

Figures 16 and 17 show CORAR mounted on the DeHavilland Beaver aircraft that we leased from Kenmore Air Seaplanes of Seattle, Washington. Figure 16 shows the radar transmitter/receiver and the data acquisition system in place in the passenger cabin of the plane. Figure 17 shows the radar antennas mounted on the starboard float of the airplane. Once again the antennas have been modified. Now, each vertically polarized, slotted-waveguide antenna was used for both transmission and reception. The antennas were mounted with $60^{\circ}$ incidence angles and one was directed forward of the perpendicular to the plane axis by $8.1^{\circ}$, the other aft at $5.9^{\circ}$. Each antenna had a $25^{\circ}$ full, one-way, half-power beamwidth in the vertical and $1.8^{\circ}$ beamwidth in the horizontal. Fore and aft antennas were alternated between scans so that every other scan came from either the fore or aft antenna. A run consisted of a total of 1000 scans collected in 6 minutes. The first 6 scans were used for calibration while the next 5 were used to define the system noise level. Thus 989 scans of useable data were collected on each run and by plotting these as functions of time and ground range, images (intensity maps) could be produced. If the plane flew without yaw, our $500 \mathrm{~Hz}$ sample rate allowed it to fly at $56.7 \mathrm{~m} / \mathrm{s}$ without aliasing the spectra. In practice, however, the plane did yaw so a method was established during data processing to unwrap the aliased spectra. Data were collected from flights along the Nooksack, Skagit, Sauk, Stillaguamish, Nisqually, Cowlitz, and Chehalis Rivers in western Washington state.

Yaw was obtained from the heading produced by the same AHRS that we used on the helicopter. We mounted it inside the plane just behind the rear passenger seat. We again 


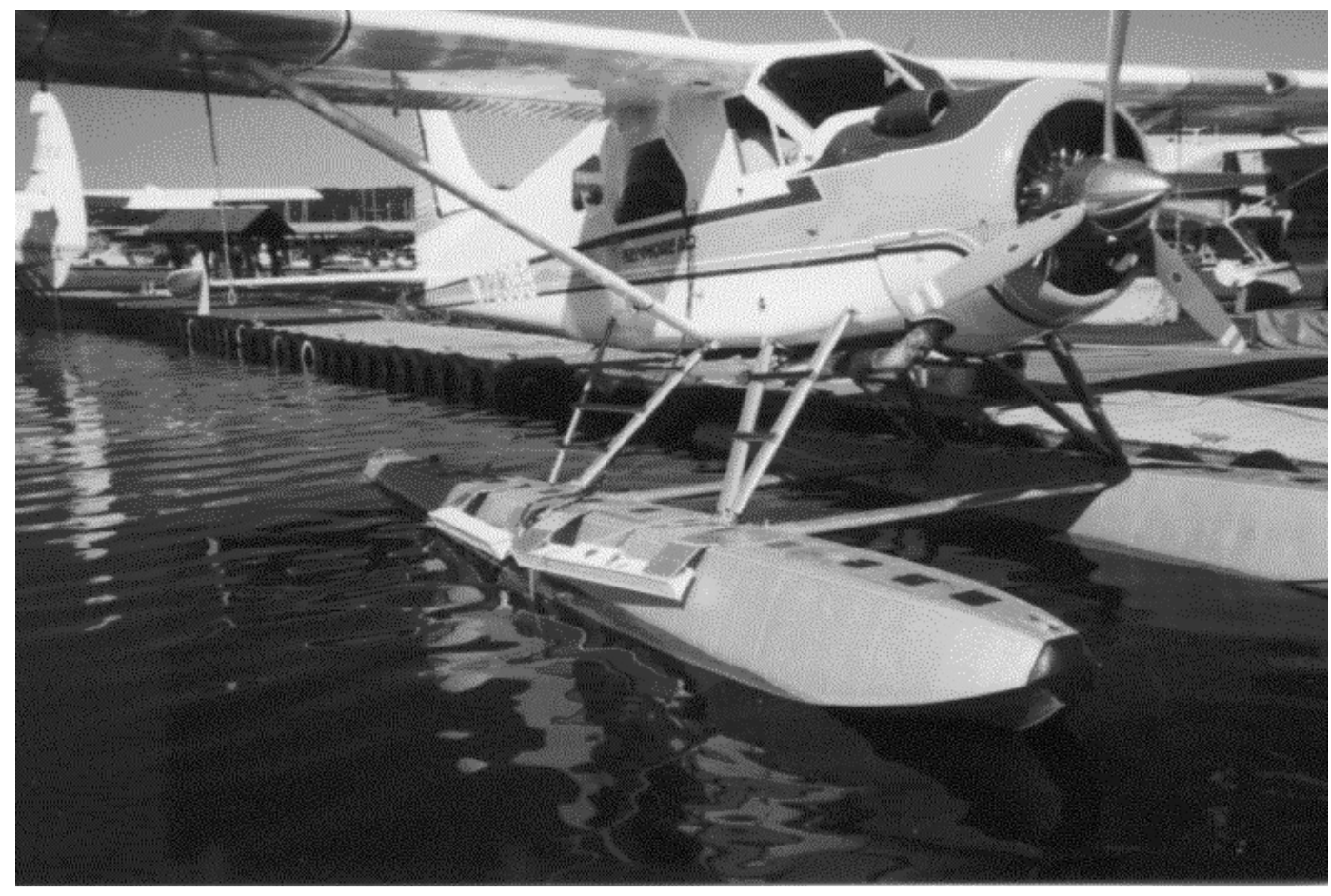

Figure 17: The DeHaviland Beaver outfitted with CORAR. The two transmit/receive antennas of the radar are mounted on the starboard float of the plane.

found that the heading from the AHRS was biased and had to be corrected using the output of our Doppler radar. The location and velocity of our flights were obtained from a differential GPS system whose satellite antenna we mounted on the roof of the aircraft and whose beacon antenna was mounted below the fusilage. We found that differential lock could be obtained except when we flew well up into the mountains.

Data processing subsequent to the flights was done in Matlab. Recorded housekeeping variables were plotted to make certain that the data had been collected in conditions acceptable for processing. The flight track, altitude, ground speed, pitch, roll, and heading as a function of time were examined. Regions with large pitch or roll were omitted from further processing. The recorded Doppler offsets were adjusted for folding of the spectrum at times when Doppler spectra were aliased. The adjusted offsets where then corrected for signal-to-noise ratio (SNR) by multiplying them by $(1+\mathrm{SNR}) / \mathrm{SNR}$ as described in detail by Plant et.al., 1998. This procedure is only important for low SNR; we omitted data where $S N R<2$. The noise level of our system corresponded to a normalized radar cross section of the surface of less than $-40 \mathrm{~dB}$ over nearly all of our illuminated surface pattern.

When corrected, measured Doppler offsets were available, they could be used to correct the yaw of the aircraft. First, we needed to know the incidence angle as a function of range. This is not straightforward to determine when the elevation of the ground varies across the swath measured by the radar footprint. We determined it to first order by using Eq.(2) with 
$V_{l}=-V \sin \theta \sin \phi$, where $\mathrm{V}$ is the plane speed. This is

$$
f_{d i}=\frac{2 V \sin \theta \sin \left(\phi_{i}-\phi_{y}\right)}{\lambda}
$$

where " $\mathrm{i}$ " is " $\mathrm{f}$ " for the fore antenna and "a" for the aft, $\phi_{i}$ is horizontal look-direction of antenna "i" minus the perpendicular to the plane, $\phi_{y}$ is aircraft yaw, and $\lambda$ is the microwave length. Substituting the measured Doppler offsets, $f_{d f}$ and $f_{d a}$, from the fore and aft antennas for $f_{d i}$ and approximating $\sin \left(\phi_{i}-\phi_{y}\right)$ by $\phi_{i}-\phi_{y}$, we obtain

$$
V \sin \theta=\frac{\lambda\left(f_{d f}-f_{d a}\right)}{2\left(\phi_{f}-\phi_{a}\right)}
$$

Note that the yaw drops out in this approximation. In our farthest range bins, $\sin \theta$ was always near 0.975 . Thus $\mathrm{V}$ could be determined from the far range bins and then $\sin \theta$ as a function of range $\left(f_{d f}\right.$ and $f_{d a}$ depend on range) could be determined.

One further calculation had to be made before yaws could be corrected. Antenna azimuth angles had to be adjusted for the effects of aircraft pitch, $\alpha$. The corrected azimuth angles for the antennas, $\phi_{c i}$, are given by

$$
\phi_{c i}=\tan ^{-1}\left[\sin \phi_{i} /\left(\cos \left(\phi_{i}-\alpha / \tan \theta\right)\right]\right.
$$

These corrected azimuth angles were then substituted for $\phi_{i}$ in Eq.(12) to compute the expected Doppler shifts $f_{d i}$. The average computed values for the 70 farthest range bins were compared with the corresponding measured values and the yaw was iteratively adjusted until the measured and computed values of $f_{d i}$ agreed within a small tolerance. The results are corrected yaw values that are more accurate than those measured by the compass. We found that the corrected yaw usually differed from the measured one by less than about $15^{\circ}$.

Given the corrected values of yaw, along with the azimuth angles of the antennas, the plane velocity, and the incidence angles, it was possible to shift the data from the aft antenna to obtain for each range bin the value of $f_{d a}$ that was measured at the spot closest to that at which $f_{d f}$ was measured. The necessary procedure turned out to be quite complex. It was basically to use the flight geometry to shift the data from the aft antenna by different amounts for different range bins such that the surface spot closest to the measurement location of the fore antenna was obtained. Because of the uncertainties in the recorded flight parameters, however, this calculation did not always produce the optimum choice. It was eventually found that the chosen shift of the aft antenna data could be adjusted slightly by minimizing the difference in SNR between the fore and aft antennas. This adjustment gave results that clearly indicated that both antennas were viewing the same spot.

Using these collocated values of fore and aft Doppler shifts, improved values of $\mathrm{V}$ and $\sin \theta$ could be obtained as before but using the following more exact equation:

$$
V \sin \theta=\frac{\lambda\left(f_{d f}-f_{d a}(\text { shift })\right)}{2\left(\sin \left(\phi_{c f}-\phi_{y}\right)-\sin \left(\phi_{c a}-\phi_{y}(\text { shift })\right)\right.}
$$

where (shift) indicates shifted data from the aft antenna. Ground speeds obtained from the result of this equation in far range bins agreed very well with those measured by the GPS. 
This then allowed the computation of a current component in the direction of each antenna at every resolution cell in a river. First the Doppler shifts produced by river surface currents had to be obtained from the total Doppler shifts by subtracting the Doppler shift that a stationary scatterer would have in the aircraft frame of reference. This was accomplished by using the SNR to determine where the river was located. The top panel of Figure 18 shows an image of the Skagit River produced by CORAR; the river is very obvious due to its very low SNR. The black vertical stripes are times when the aircraft pitch or roll was too large. Doppler shifts coming from resolution cells with high SNR were fit by interpolation, interpolating also across regions where the SNR was low, usually streamflow features. Subtraction of this interpolated Doppler shift, $f_{\text {dio }}$ from the measured ones, $f_{d i}$ then provided a Doppler shift due to scatterer motion at each resolution cell. From these, the line-of-sight velocities, $V_{l}$, could be computed for each antenna from the following equation:

$$
V_{l i}=\frac{\lambda\left(f_{d i}-f_{d i o}\right)}{2 \sin \theta}
$$

where, as usual, $i$ is either $f$ or $a$.

Our initial hope was that the complete current vector could be computed from these two measured components as we had done from riverbanks (See Section 3.3). The angle between the two components was so small and the uncertainty of $f_{d i}$ and $f_{d i o}$ so large, however, that the error in this calculation was unacceptable. Therefore a method was developed where the course of the river was determined from the intensity image by hand. The two measured current components could then be averaged, yielding $\left\langle V_{l}\right\rangle$ and the angle, $\phi_{o}$, between the mean horizontal look-direction of the antennas and $+v$ axis could be determined. Then $u$ was given by Eq.(4):

$$
u=\frac{<V_{l}> \pm c}{\sin \theta \sin \phi_{o}}
$$

Clearly, if $\phi_{o}$ is too small, this procedure will produce large errors in $u$. For these aircraft measurements, $f_{d i}$ was computed from the first moment of the Doppler spectrum, which is severely broadened by plane motion. Thus we have no way of determining the effects of the Bragg wave phase speed so we ignored "c" in the above equation.

The above processing procedures were applied to the data collected during flights. Figures 18 and 19 show results obtained from Run 169, a flight along the Skagit River on October 1, 1999. The images in Figure 18 clearly show that river surface velocities could only be determined infrequently, three times over the whole $18 \mathrm{~km}$ reach displayed. Figure 19 is a plot of the cross-river profile of current obtained from the section of reach near $5 \mathrm{~km}$. Clearly the current could be measured only part way across the river. This was typical of other results we obtained from these flights. We were not able to compare any currents measured by the airborne CORAR with in situ measurements.

\section{INSAR Measurements of River Surface Currents}

We consider now whether river surface currents could also be measured from a satellite using microwave techniques. The most obvious candidate for making such measurements 


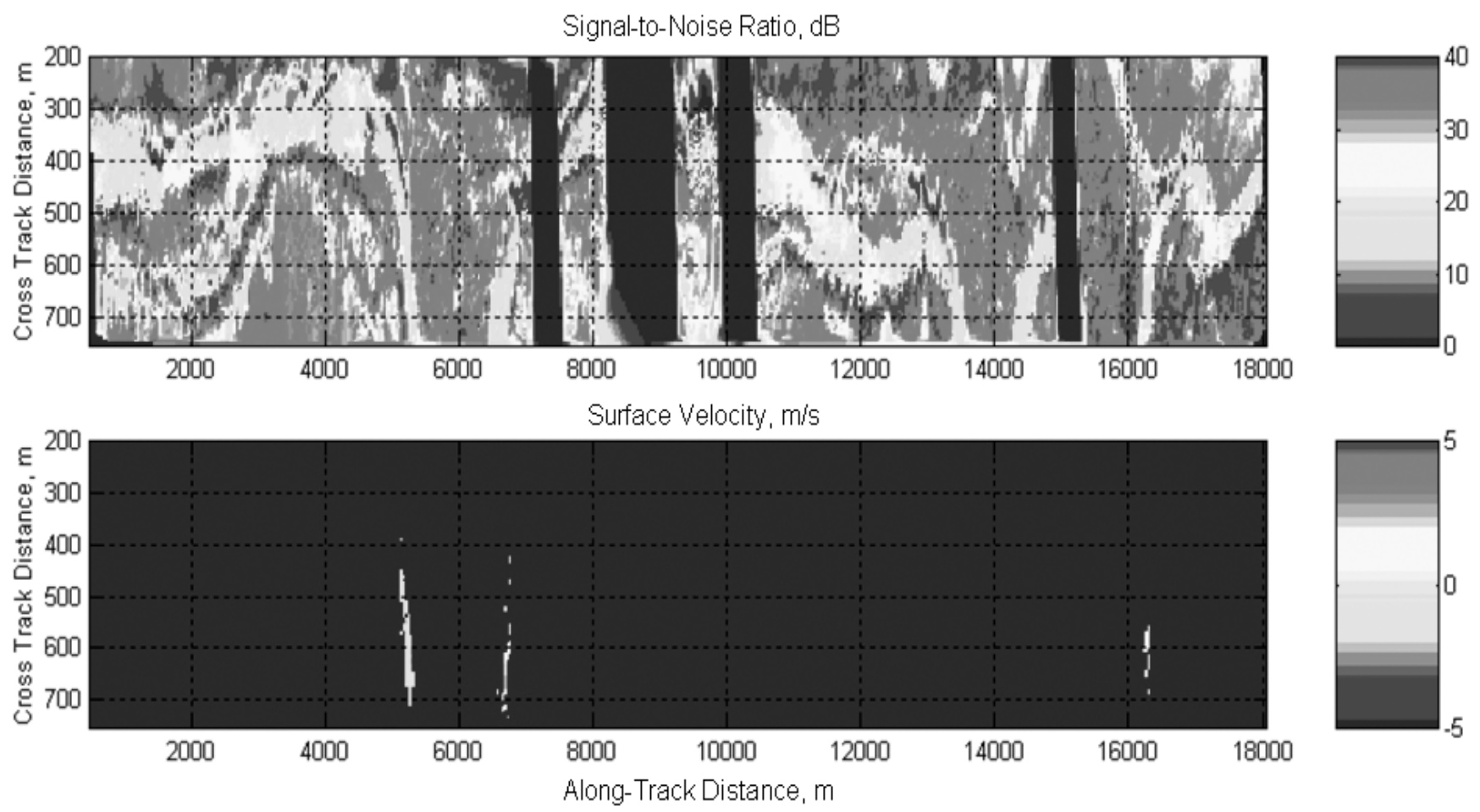

Figure 18: Intensity image (top), current velociy map with currents toward the radar being negative (bottom) for Run 169 along the Skagit River on October 1, 1999. Color bars give the signal-to-noise ratio in $\mathrm{dB}$ in the top panel and the current velocity in $\mathrm{m} / \mathrm{s}$ in the bottom panel. 


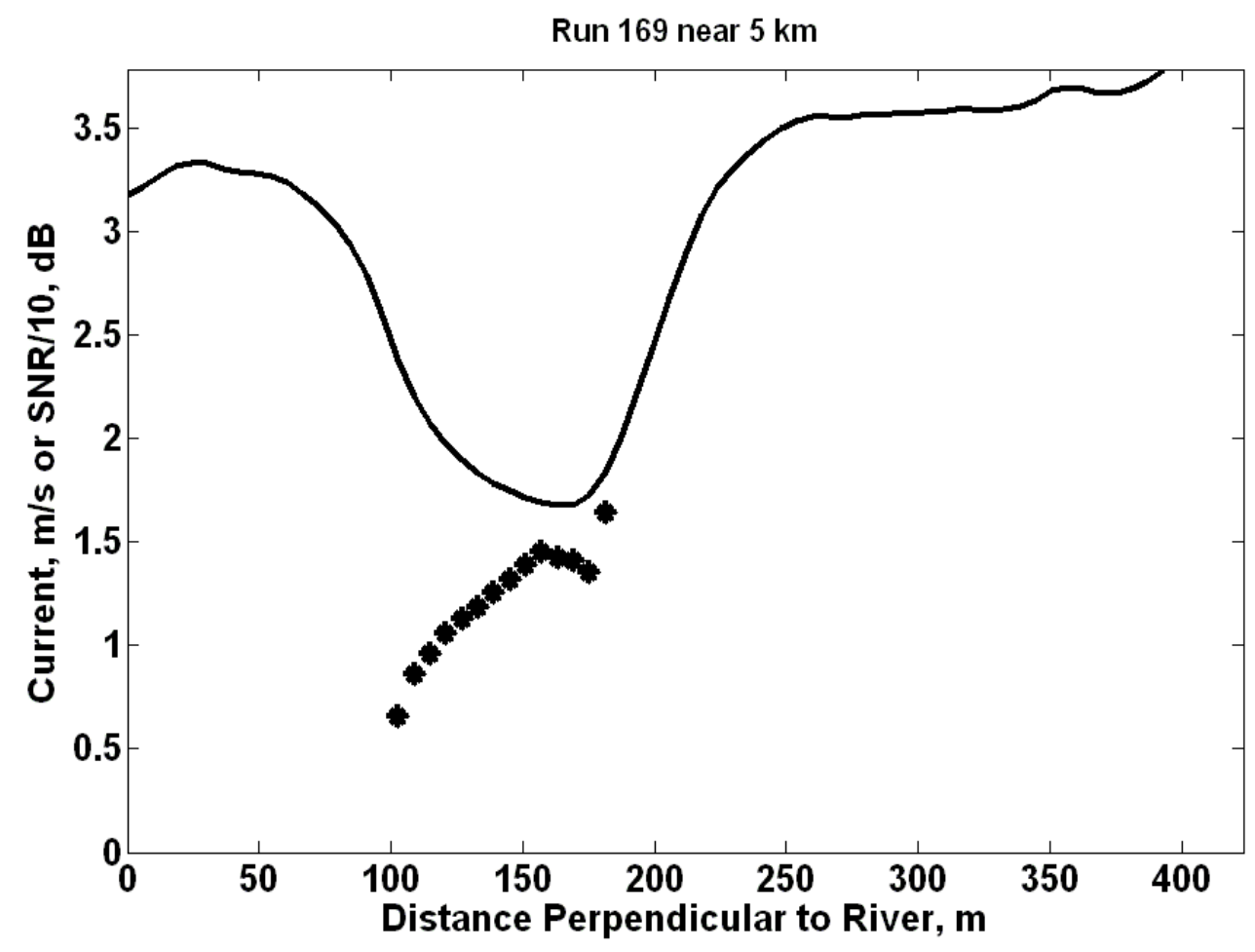

Figure 19: Current profile (asterisks) and SNR level (line) for the section of Run 169 near 5 $\mathrm{km}$.

is interferometric synthetic aperture radar (INSAR) because of its high resolution, dayand-night, all-weather, imaging capabilities. We begin by briefly outlining the synthetic aperture radar (SAR) principle and then discussing INSAR. These considerations reveal several limitations on the use of INSAR for measuring river surface currents either from aircraft or from satellites.

\subsection{Basics of SAR}

Microwave signals are normally transmitted by a highly directional antenna whose beamwidth $\Phi$ in radians is approximately given by

$$
\Phi=\frac{\lambda}{D}
$$

where $\mathrm{D}$ is the antenna diameter. Imaging radars are usually flown either in airplanes or spacecraft with the antenna pointed perpendicular to the direction of flight as shown in Figure 20. Image resolution is determined by different radar properties perpendicular and parallel to the flight track. Resolution $\rho_{r}$ in the range direction is determined by the width of the transmitted pulse. As the pulse sweeps across the surface, the power received by the antenna varies according to the scattering properties of the surface area within the pulse. In this way, an image of the surface in the range, or cross track, direction is produced. The second dimension of the image, the azimuth, or along track, dimension, is produced by the 

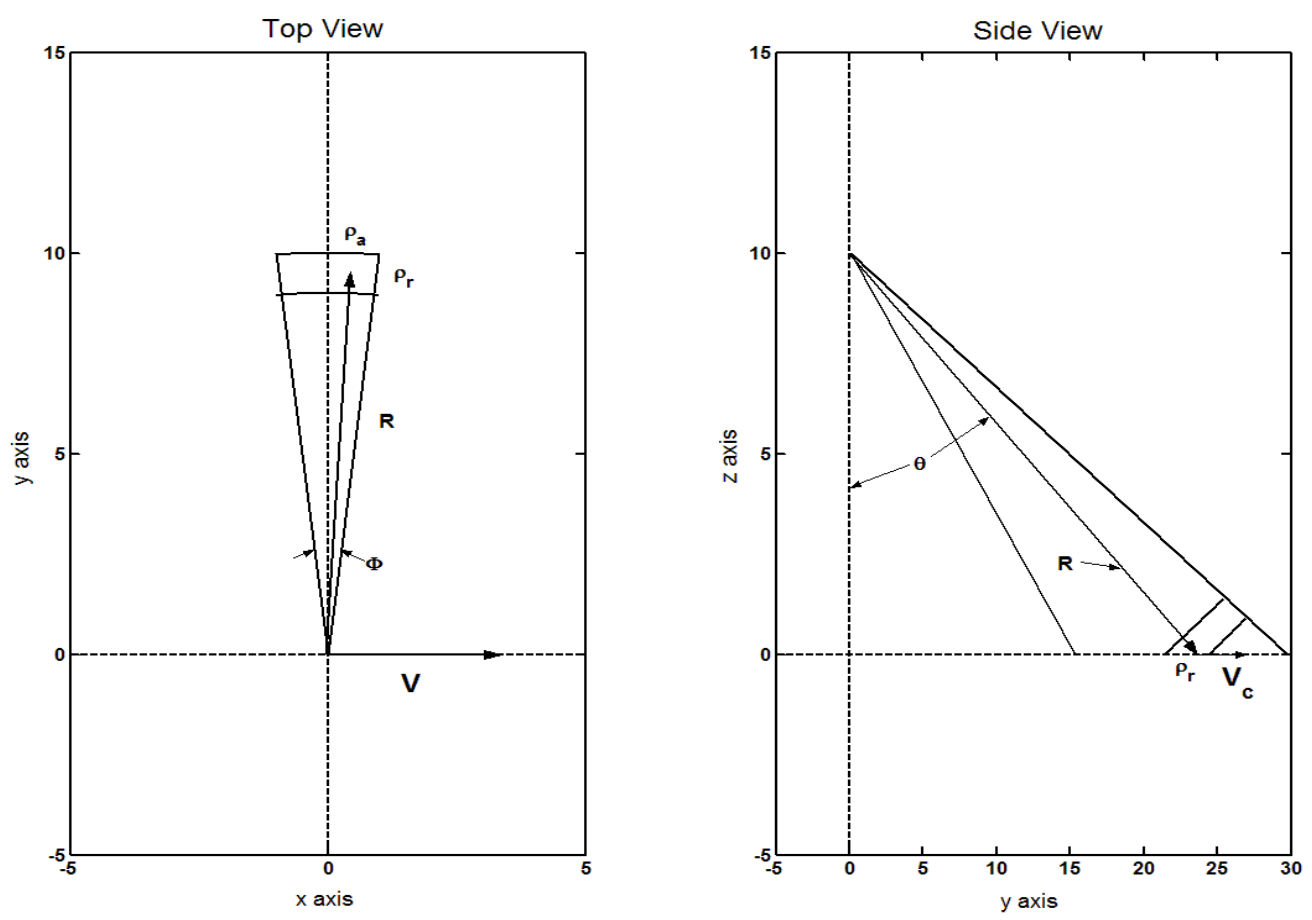

Figure 20: Standard SAR Geometry

motion of the plane or satellite. Thus in Figure 20 successively different strips in the y direction are imaged as the plane moves along the $\mathrm{x}$ axis. For a radar like CORAR that uses the antenna beamwidth to produce resolution $\rho_{a}$ in this dimension, we clearly have

$$
\rho_{a}=\frac{\lambda R}{D}
$$

As the range $\mathrm{R}$ increases, the resolution of the image in the $\mathrm{x}$ direction increases and the image loses detail in this dimension. This is why Real Aperture Radars (RARs) cannot resolve objects smaller than a kilometer or so from space. To obtain better resolution would require a prohibitively large antenna.

SAR techniques were developed to overcome this problem. SARs must be coherent so that they can determine the Doppler shifts induced in the backscattered signal due to the relative motion of the plane and the surface (Harger, 1970). Given this ability, SAR uses the fact that the Doppler shift produced by any point on the surface as it travels through the antenna beam (due to the plane's motion) is easy to describe if the point is stationary on the surface. Eq.(2) for the Doppler frequency shift $f_{d}$ produced by relative motion of the antenna and target is

$$
f_{d}=-\frac{2 V_{l}}{\lambda}
$$

A point on the surface at the tip of the vector $\vec{R}$ in Figure 20 appears to be moving in the -x direction with a velocity $-\vec{V}$ to observers in the plane. Its line-of-sight component along 
$\vec{R}$ is, to a good approximation,

$$
V_{l}=-\frac{V x}{R} .
$$

Therefore the Doppler shift is

$$
f_{d}=\frac{2 V x}{\lambda R}
$$

When $f_{d}=0$, the target is directly perpendicular to the plane so we have located its azimuthal position. Now we must determine the accuracy of this location, that is, the azimuthal resolution of the image.

The Doppler shift $f_{d}$ is determined from the spectrum of the received signal. But a given point on the surface will only stay in the antenna beam for a finite time T. Thus $f_{d}$ cannot be determined more accurately than $1 / \mathrm{T}$. The most accurately $\mathrm{x}$ can be determined for a stationary scene, the azimuthal resolution $\rho_{a s}$, can then be determined from Eq.(22) by letting $f_{d}$ and $\mathrm{x}$ vary slightly:

$$
\Delta f_{d}=\frac{2 V \Delta x}{\lambda R}=\frac{1}{T}
$$

Noting that $\rho_{a s}=\Delta x$ we have

$$
\rho_{a s}=\frac{\lambda R}{2 V T}
$$

But to a good approximation $V T=\Phi R$ which, by Eq.(18), is $V T=\lambda R / D$. Substituting this into Eq.(22) yields

$$
\rho_{a s}=\frac{D}{2} .
$$

For stationary scenes, the SAR technique produces azimuthal resolutions that are half the diameter of the antenna, much smaller than those that can be produced by RARs with the same size antenna (see Eq.(19)). Furthermore the SAR azimuthal resolution does not depend on range so the technique is applicable at the very large heights of satellite-borne instruments.

The SAR technique has a problem, though, if the target on the surface is moving. In this case, the Doppler shift produced by the relative motion of the plane and the moving target is given by (See Figure 20)

$$
f_{d}=\frac{2 V x}{\lambda R}-\frac{2 V_{c} \sin \theta}{\lambda}
$$

where $V_{c}$ is the component of scatterer velocity in the y direction. Now when $f_{d}=0$,

$$
x=\frac{R V_{c} \sin \theta}{V} .
$$


The $\mathrm{x}$, or azimuthal, position of a moving target is displaced in a SAR image by an amount proportional to its line-of-sight velocity. This phenomenon is very observable: trains moving perpendicular to the flight direction appear to be displaced from their tracks in SAR images.

The situation is even more complex if the velocity of the target is not constant (Hasselmann et.al., 1985). We can relate $\Delta x$ to the velocity variation of the target $\Delta V_{c}$ using Eq.(27). In turn, $\Delta V_{c}$ can be related to the width of the Doppler spectrum $\Delta f_{d s}$ measured from a stationary platform using Eq.(26) with $V=0$ :

$$
\Delta f_{d s}=\frac{2 \Delta V_{c} \sin \theta}{\lambda}
$$

We define the coherence time of the surface to be $\tau_{c}=1 / \Delta f_{d s}$ and the resolution of the moving scene to be $\rho_{a m}=\Delta x$. Using these definitions along with Eqs. (27) and (28), we get

$$
\rho_{a m}=\frac{\lambda R}{2 V \tau_{c}}
$$

Since the resolutions $\rho_{a s}$ and $\rho_{a m}$ are related to standard deviations of the independent random variables $\Delta f_{d}$ and $\Delta f_{d s}$, the total azimuthal resolution $\rho_{a}$ of a SAR image is given by

$$
\rho_{a}=\sqrt{\rho_{a s}^{2}+\rho_{a m}^{2}} .
$$

This shows that $\rho_{a m}$ is the best resolution that can be obtained for a scene that is in motion.

Widths of rivers can be obtained directly from SAR images. The accuracy of the determination of width will be limited by the range and azimuthal resolutions of the system, $\rho_{r}$ and $\rho_{a s}$. The resolution of the moving water surface, $\rho_{a m}$ does not usually play a role in this determination since backscatter from the river is generally much smaller than that from the banks so that the stationary scatterers dominate the signal from any cell containing both land and water returns.

\subsection{INSAR}

In standard SAR processing, the fields received by the antenna from each resolution cell are multiplied by their complex conjugates to yield the power received from each cell. The field image is called the "phase image" while its magnitude squared is the standard SAR image. It is possible, however, to add a second antenna to the SAR being flown and to multiply the phase image from one by the complex conjugate of the phase image of the other, registered to observe precisely the same scene. This is called interferometric SAR and yields information about the relative phases of the signals received by the two antennas (Zebker and Goldstein, 1986; Goldstein and Zebker, 1987; Goldstein et.al., 1989). (Here we assume transmission and reception occur at both antennas. It is possible to use one antenna only as a receiver but the situation is slightly more complex.) The antennas may be separated in any one of the three spatial dimensions. Here we consider separation in the $\mathrm{x}$ and $\mathrm{y}$ dimensions shown in Figure 20. The modified situation is depicted in Figure 21.

In this figure we indicate that the surface is not necessarily at $z=0$ but rather at $z=\gamma(x, y)$. Clearly, at an earlier time $\tau=\Delta x / V$, the tip of $\overrightarrow{R_{2}}$ will have coincided with 

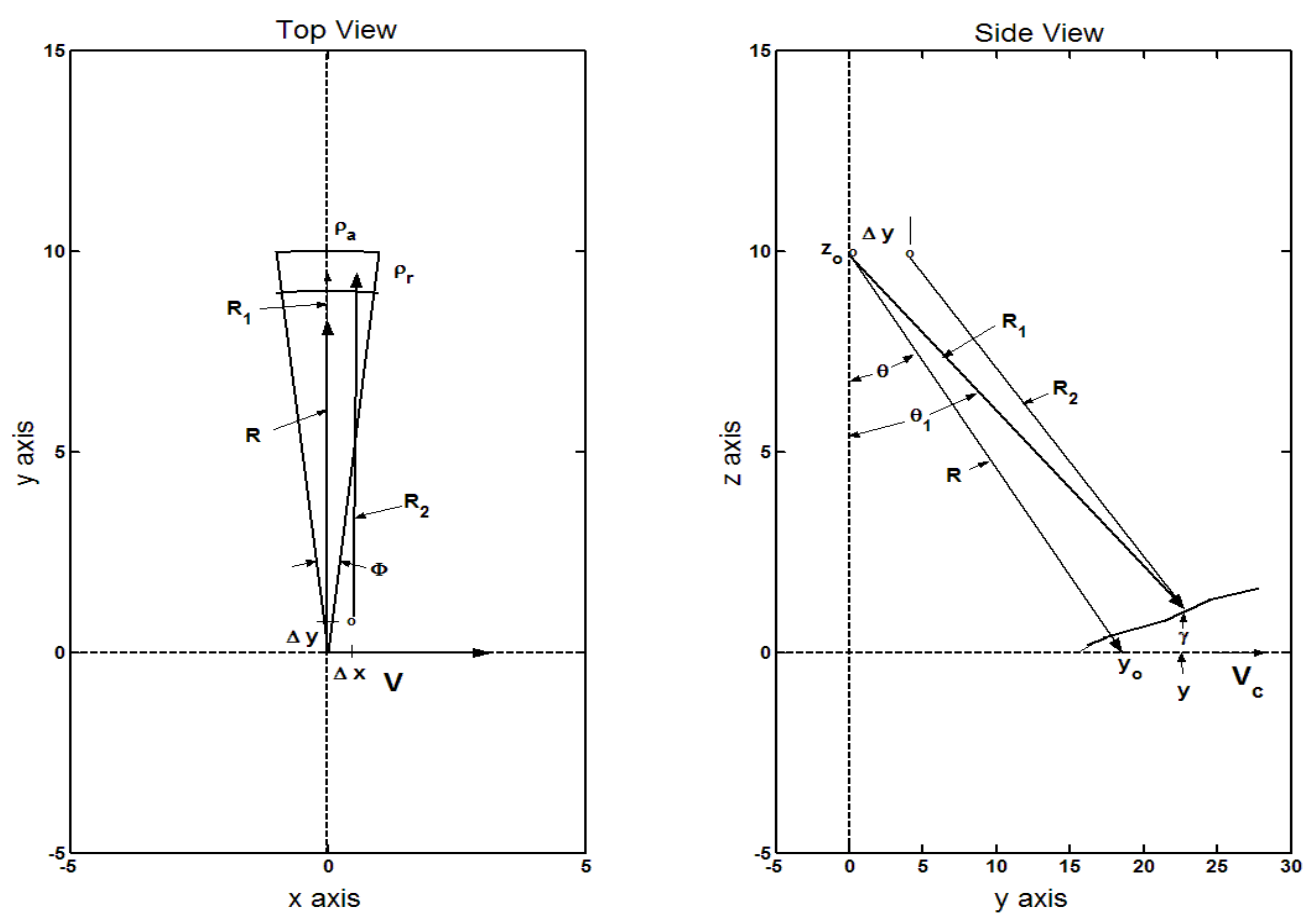

Figure 21: Interferometric SAR Geometry

the present location of the tip of $\vec{R}_{1}$. Note that in this time interval, a target moving horizontally away from the antenna at speed $V_{c}$ will move a distance $V_{c} \tau$. Since the time history of the pulse sweeping across the surface yields only the magnitude of the range, it cannot distinguish $\vec{R}$ from $\overrightarrow{R_{1}}$ if $R=R_{1}$. The phases of the signal received at antennas 1 and 2 are given by $2 k R_{1}$ and $2 k R_{2}$, where $k=2 \pi / \lambda$. If $\mathrm{y}$ is the position of the target at time $t=0$ then the range $R_{2}$ at (the earlier) time $t=\tau$ is given by

$$
R_{2}=\sqrt{\left(z_{o}-\gamma\right)^{2}+\left(y-V_{c} \tau-\Delta y\right)^{2}}
$$

This may be approximated by

$$
R_{2}=R_{1}-\sin \theta_{1}\left(V_{c} \tau+\Delta y\right)
$$

by ignoring squares of the small (relative to $z_{o}$ ) quantities $V_{c} \tau$, and $\Delta y$ and expanding the square root. Here $\theta_{1}$ is the incidence angle of $R_{1}$. Thus, the phase difference, $\Delta \phi$ between the signals received by antennas 1 and 2 when they view the same point on the surface is

$$
\Delta \phi=2 k\left(R_{1}-R_{2}\right)=2 k \sin \theta_{1}\left(V_{c} \tau+\Delta y\right) .
$$

Eq.(33) will yield $V_{c}$ if $\sin \theta_{1}$ can be determined since $k, \Delta y$, and $\tau$ are known and $\Delta \phi$ can be obtained from the interferometric SAR image.

If the surface is flat, $\sin \theta_{1}=\sin \theta$ and $\sin \theta_{1}$ is known from measurements of range and altitude. In this case, it is simplest to let $\Delta y=0$ and use an along-track INSAR. In the more general case where the surface elevation varies, $\theta_{1}$ is no longer equal to $\theta$ and we must 
use $\Delta y$ to determine $\sin \theta_{1}$. Consider a measurement of $\Delta \phi$ at the bank of the river, call it $\Delta \phi_{b}$. Since the bank is not moving, $\Delta \phi_{b}$ directly yields $\theta_{1}$ at the bank, call it $\theta_{b}$. Then from Eq.(33):

$$
\sin \theta_{b}=\frac{\Delta \phi_{b}}{2 k \Delta y}
$$

Assuming that $\gamma$ at the riverbank is nearly the same as it is on the river,

$$
R_{b} \cos \theta_{b}=R \cos \theta_{1}
$$

where $R_{b}$ is the range to the river bank. Thus,

$$
\sin \theta_{1}=\sqrt{1-\left(\frac{R_{b}}{R}\right)^{2}+\left(\frac{R_{b} \Delta \phi_{b}}{2 R k \Delta y}\right)^{2}}
$$

is known at all points on the river, that is, at all ranges $\mathrm{R}$ that are within the river. Therefore $V_{c}$ can be determined as a function of distance across the river:

$$
V_{c}=\frac{\Delta \phi /\left(2 k \sin \theta_{1}\right)-\Delta y}{\tau} .
$$

where $\Delta \phi$ and $\sin \theta_{1}$ depend on y. Knowing $V_{c}$ and the direction of river flow from the image, one can compute $u$ in the same manner as in our CORAR measurements.

\subsection{Limitations and Uncertainties of the Technique}

Several limitations must be imposed on various parameters to implement the technique successfully. Furthermore, some limitations are imposed on other parameters by the technique itself. These limitations either restrict system parameters or cause uncertainties to exist in the measurements. A more detailed discussion of some of these limitations than has been given here may be found in Romeiser and Thompson (2000) and Romeiser et al., 2002.

\section{The time $\tau$ must be short.}

The signal must not change form significantly during the time between measurements by the front and rear antennas. This means that we must have

$$
\tau<\tau_{c}=\frac{1}{\Delta f_{d s}}
$$

The targets that INSAR sees on the water surface are the short waves travelling toward

and away from the antenna that satisfy the Bragg scattering condition. The Bragg wave is known in the absence of wind to travel at a speed given by

$$
c=\sqrt{g / k_{b}+T k_{b}}
$$

where $g=981 \mathrm{~cm} / \mathrm{s}^{2}$ is gravitational acceleration, $T=74 \mathrm{~cm}^{3} / \mathrm{s}^{2}$ is surface tension divided by water density, and $k_{b}=2 \pi / \lambda_{b}$ is the Bragg wavenumber. 
Measurements (See Figures 3 and 4) show that

$$
\Delta f_{d s} \approx f_{b}=c / \lambda_{b}
$$

Thus we require that

$$
\tau<\frac{\lambda_{b}}{c}=\frac{\lambda}{2 c \sin \theta}
$$

Figure 22a shows this limitation on $\tau$ as a function of radar wavelength assuming that $\theta=45^{\circ}$; the corresponding limitation on the antenna separation $\Delta x$ is obtained by multiplying $\tau$ by the velocity of the platform.
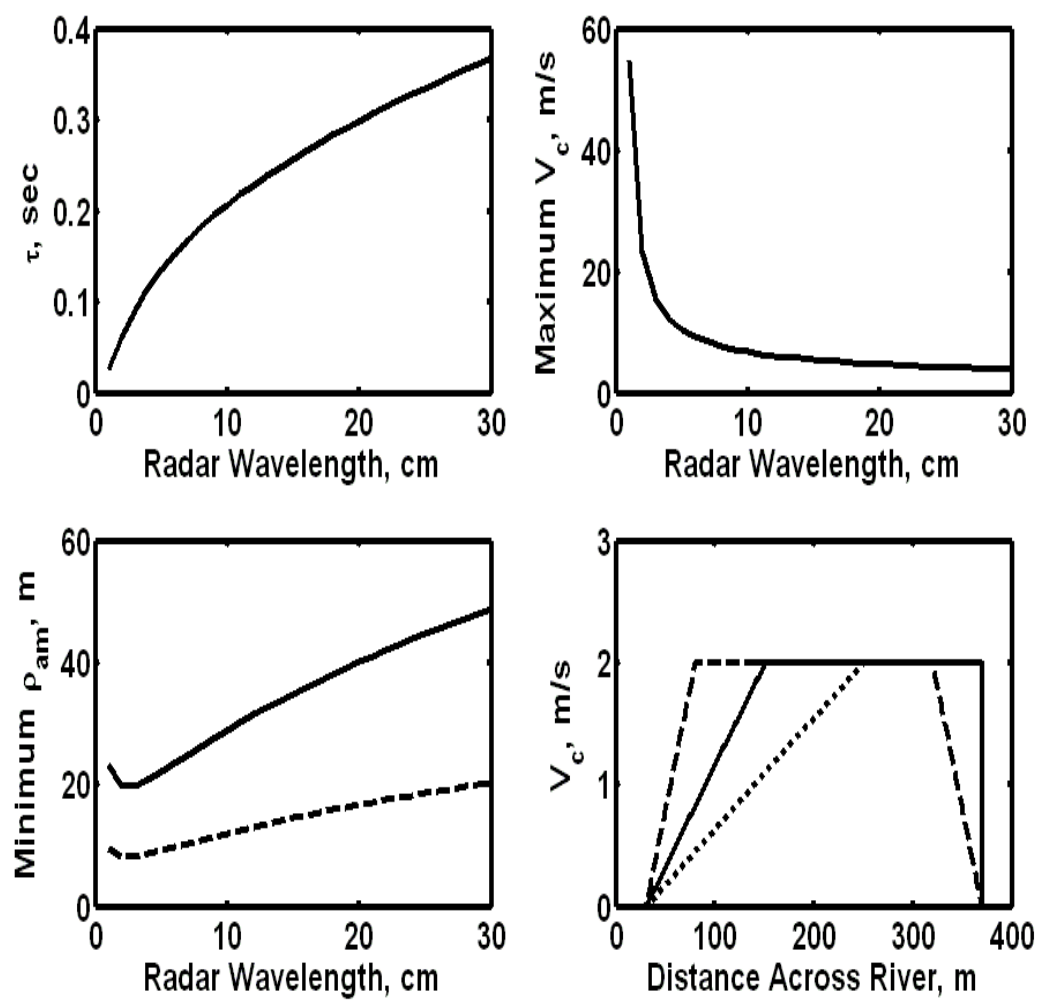

Figure 22: INSAR parameters related to rivers. In panels a, b, and c, $\theta=45^{\circ}$. a) Maximum possible time lag between antennas for different radar wavelengths. b) Maximum measureable surface velocity in the range direction versus radar wavelength assuming that $\rho_{r}=1$ m. c) Minimum azimuthal resolution versus radar wavelength. The solid line is for $\mathrm{R} / \mathrm{V}=$ $120 \mathrm{sec}$ (spacecraft) and the dashed line is for $\mathrm{R} / \mathrm{V}=50 \mathrm{sec}$ (aircraft). d) Velocity profiles across the river. The dashed line is the true profile, the solid line is produced by INSAR with $\mathrm{R} / \mathrm{V}=50 \mathrm{sec}$, and the dotted line is produced by INSAR with $\mathrm{R} / \mathrm{V}=120 \mathrm{sec}$.

\section{The target must not move out of the resolution cell in time $\tau$}


If the target travels too fast, it will leave the resolution cell in the time between observations of the cell by the two antennas. Since the system only senses the target velocity in the range direction, this limitation is

$$
V_{c} \tau \sin \theta<\rho_{r}
$$

To obtain the minimum azimuthal resolution, $\tau$ should be chosen to be as large as possible. Substituting the maximum value of $\tau$ from Eq.(41) into this, we find that we must require that

$$
V_{c}<\frac{2 c \rho_{r}}{\lambda}
$$

The maximum value of $V_{c}$ is plotted in Figure $22 \mathrm{~b}$ for $\rho_{r}=1 \mathrm{~m}$. Note that we can increase this maximum either operating at higher microwave frequencies or at larger range resolutions.

\section{A minimum azimuthal resolution exists for rivers.}

As pointed out above, the minimum azimuthal resolution that can be obtained when imaging the flowing river is $\rho_{a m}$ given by Eq.(29). From aircraft, typical values of $R / V$ are around $50 \mathrm{sec}$ while from space they are closer to $120 \mathrm{sec}$. Using these values of $\mathrm{R} / \mathrm{V}$ in Eq.(29) and setting $\tau_{c}$ equal to the maximum value of $\tau$ from Eq.(41) yields the plots shown in Figure 22c for the minimum azimuthal resolution over the water as a function of microwave length for $\theta=45^{\circ}$. The solid lines are for the higher, space-based $\mathrm{R} / \mathrm{V}$ ratio. The minimum in this plot near a radar wavelength of $2 \mathrm{~cm}$ suggests $\mathrm{Ku}$ band, not far from our X-band system, as an operating frequency for the INSAR. As our airborne results show, a river should be imaged when flying nearly perpendicular to it so that the maximum along track phase shift will be produced by the current. In this situation, $\rho_{a m}$ is the limit of how closely spaced independent measurements of $V_{c}$ can be made. Stated differently, the true river current as a function of position across the river will be smoothed by a filter of width $\rho_{a m}$.

\section{Moving scatterers will be shifted out of the river.}

Recall that Eq.(27) says that a target having a component of velocity in the range direction will be displaced from its true along-track position in a SAR image. This means that targets sufficiently close to a riverbank can be shifted to a position on the bank if their velocity is large enough. When this happens, the SAR will generally no longer see a phase shift due to velocity at this bank location because the signal from the bank itself will overwhelm the signal displaced from the river. This means that velocity profiles across the river will be distorted from their true appearance by the SAR imaging process. Figure 22d shows this effect for a flight directly across a river flowing with $V_{c}=2 \mathrm{~m} / \mathrm{s}$ at the center and being imaged with $\theta=45^{\circ}$. The figure shows the true current profile as a dashed line, that produced with $R / V=50 \mathrm{sec}$ as a solid line, and that produced with $R / V=120 \mathrm{sec}$ as a dotted line. Obviously, the problem will be greater for spaceborne application than for airborne flights. In principal, much of this distortion can be removed because $V_{c}$ is measured 
so the apparent target along-track position can be corrected. A gap in the profile will always exist near the bank where the scatterers are being shifted out of the river, however, since $V_{c}$ will be lost here due to the large signal from the bank.

\section{The measured velocity will be a weighted mean near the surface.}

The basic outline of the INSAR technique presented here could not show that the measured velocity is really a weighted mean velocity near the water surface. In fact, this velocity is obtained from the centroid of the Doppler spectrum, as it is in our airborne measurements, and several factors can cause this not to be an accurate indication of the surface velocity (Thompson and Jensen, 1993). One factor is modulation of the Bragg waves by longer waves. This can be important on the ocean where much longer waves usually exist. On rivers, however, we do not expect this normally to be a problem. A larger source of concern is that the Doppler spectrum is always made up of a combination of the spectra of Bragg waves travelling toward and way from the antenna and their relative amplitudes are not well known. As discussed in Section 2, this can cause uncertainties in the measured surface velocity of up to $\pm c$. At $\mathrm{Ku}$ band, this is about $\pm 30 \mathrm{~cm} / \mathrm{s}$. As with our airborne CORAR measurements, it is difficult to see how algorithms such as we used for measurements from stationary platforms can be implemented in the INSAR technique. These require Doppler spectra at a single location without broadening due to plane motion. INSAR, like CORAR, does not normally produce such Doppler spectra.

\section{Riverbanks are not at the water level.}

Riverbanks slope upward from the level of the water in the river. Thus our assumption that the height of the river is the same as that of the water level is not strictly correct. If the height of the bank is known, this can be easily handled by adding the difference $\Delta \gamma$ to the right side of Eq.(35). Generally this will not be possible. Therefore an error of size $\Delta \gamma / R$ will exist in our determination of $\cos \theta_{b}$. This is very small for aircraft operation and entirely negligible for operation from space.

\section{Conclusions}

This study has investigated the microwave measurement of river surface velocities from ground, air, and space. These techniques and their possible usefulness have been summarized in Table 1. We draw the following conclusions from the results of this study.

1. Measurements of river surface velocity from bridges, cableways, and riverbanks have been shown to be accurate to within about $10 \mathrm{~cm} / \mathrm{s}$ when compared with in situ measurements. These offer the possibility of long-term, non-contact, automatic measurement of river flow to supplement standard river stage measurements. Simple, inexpensive, continuous-wave, homodyne systems can only measure the surface velocity at one point in the river and under low-flow, low-wind conditions may not receive sufficient signal to be able to measure surface velocity. More expensive hetrodyne systems such as RiverRad do not suffer this problem as 
severely, although even they cannot measure surface velocity when the surface is perfectly flat.

2. For airborne or spaceborne measurements, river surface currents must have an appreciable component in the antenna look direction in order to be detectable above normal uncertainties in measured Doppler offsets. This contrasts with the situation for ground-based measurements where currents flowing as much as $75^{\circ}$ from the antenna look direction are easily detectable. The best way we have found to ensure a very strong signal in airborne settings is to operate from a helicopter whose downwash generates surface roughness.

3. Under natural conditions, detectable power levels are not usually backscattered from river surfaces and when they are, are not usually present across the entire width of a river. This statement applies specifically to our CORAR measurements where the limit of detection was a normalized radar cross section of $-40 \mathrm{~dB}$. This problem made it impossible to obtain discharges from our airborne measurements and will probably limit the ability of INSAR either from air or space on many rivers.

4. The change of incidence angle that results from changes in land elevation near rivers and from the downhill flow of rivers can be determined from the observed Doppler shifts produced by the land surrounding rivers. For INSAR this will require an antenna offset in the cross-track direction as well as the one in the along-track direction necessary for current detection.

5. Resolution across the river will be reduced for INSAR operation relative to its resolution on land due to motion effects. Subresolution velocity variations, which are always present on water surfaces, will reduce the coherence time of the backscatter relative to those for fixed targets. This increases SAR's azimuthal resolution, which will usually have a large component in the cross-river direction in river current measurements.

6. Currents along one side of a river will not be measured by INSAR due to motion effects in the imaging. This will be a more severe problem from space than from aircraft due to the larger R/V ratios that usually apply in space. In both cases, it will be a more severe problem for narrow rivers where the loss of information could exceed 50\%. In many cases, though, this problem may be no more severe than that encountered with acoustic techniques, which typically cannot measure near the riverbanks.

\section{Acknowledgements}

The authors would like to thank Robert Contreras who operated CORAR during many of the aircraft flights. We are also grateful to Chris Siani for his help in constructing and mounting many of the systems in the field. Justin Huff and Nathan Kohagan were very instrumental in developing software used in RiverScat and we are grateful for that. We are also grateful to Kurt Spicer of the USGS for providing the comparison stage and surface velocity data. The first author would especially like to thank the members of the USGS Hydro21 committee, 
John Costa, Nick Melcher, Eugene Hayes, Ralph Cheng, Peter Haeni, and Kurt Spicer, for their help, encouragement, and support. This work was supported by the USGS through Cooperative Research Agreements 01-WRAG-0031 and 03-ERAG-0061, by NASA through Grant number NAG5-7550, and by NSF through Grant number EAR-0106391.

\section{References}

Bass, F.G., I.M. Fuks, A.I. Kalmykov, I.E. Ostrovsky, and A.D. Rosenberg, Very high frequency radiowave scattering by a disturbed sea surface. Part II: Scattering from an actual sea surface. IEEE Trans. Antenna and Propagation, AP-16(5), 560-568, 1968.

Braun N., M. Gade, and P.A. Lange, The effect of artificial rain on wave spectra and multipolarisation X band radar backscatter Intl. J. Remote Sens., 23(20), 4305-4323, 2002.

Contreras, R.F., and W.J. Plant, Ku-band backscatter from the Cowltiz River: Bragg scattering with and without rain, accepted by IEEE Trans. Geosci. Rem. Sensing, 2004.

Costa, J.E., K.R. Spicer, R.T. Cheng, F.P. Haeni, N.B. Melcher, E. Michael, W.J. Plant, and W.C. Keller, Measuring stream discharge by non-contact methods: A proof-of-concept experiment, Geophys. Res. Letters, 27, 553-556, 2000.

Goldstein, R.M. and H.A. Zebker, Interferometric radar measurements of ocean surface currents, Nature, 328, 707-709, 1987.

Goldstein, R.M., T.P. Barnett, and H.A. Zebker, Remote Sensing of ocean surface currents, Science, 246, 1282-1285, 1989.

Harger, R.O., Synthetic Aperture Radar Systems: Theory and Design, Academic Press, New York, pp. 109-128, 1970.

Hasselmann, K., R.K. Raney, W.J. Plant, W. Alpers, R.A. Shuchman, D.R. Lyzenga, C.L. Rufenach, and M.J. Tucker, Theory of SAR ocean wave imaging: A MARSEN view. J. Geophys. Res., 90 (C3), 4659-4686, 1985.

Melcher, N.B., J.E. Costa, F.P. Haeni, R.T. Cheng, E.M. Thurman, M. Buursink, K.R. Spicer, E. Hayes, W.J. Plant, W.C. Keller, and K. Hayes, River discharge measurements by using helicopter-mounted radars, Geophys. Res. Lett., 29(22), 2084, doi:10.1029/2002GL015525, 2002.

Okamoto, Y., 1999, Development of a non-contact method of measuring river depth from the air; in Environmental Hydraulics, Lee, J.H.W., Jayawardena, A.W., and Wang, Z.Y., eds., A.A. Balkema, Rotterdam, p. 965-970.

Plant, W.J., and J.W. Wright, Phase speeds of upwind and downwind traveling short gravity 
waves. J. Geophys. Res., 85 (C6), 3304-3310, 1980.

Plant, W.J. and W.C. Keller, 1990. Evidence of Bragg scattering in microwave Doppler spectra of sea return, J. Geophys. Res., 95 (C9), 16,299-16,310, 1990.

Plant, W.J., W.C. Keller, V. Hesany, and K. Hayes, Measurements of the marine boundary layer from an airship, J. Atmos. Ocean. Tech., 15, 1433-1458, 1998.

Plant, W.J., W.C. Keller, K. Hayes, and K. Spicer, Streamflow properties from time series of surface velocity and stage, submitted to J. Hydraulic Engineering, 2004a.

Plant, W.J., W.C. Keller, and K. Hayes, Simultaneous measurement of ocean winds and waves with an airborne coherent real aperture radar, accepted by J. Atmos. Ocean. Tech., 2004b.

Plant, W.J., Bragg Scattering of Electromagnetic Waves from the Air/Sea Interface, in Surface Waves and Fluxes: Current Theory and Remote Sensing. Edited by G.L. Geernaert and W.J. Plant, Kluwer Academic Publishers, 2, 1-108, 1990.

Plant, W.J., A model for microwave Doppler sea return at high incidence angles: Bragg scattering from bound, tilted waves, J. Geophys. Res, 102(C9), 21131-21146, 1997.

Plant, W.J., Microwave sea return at moderate to high incidence angles, Waves in Random Media, 13(4), 339-354, doi: 10.1088/0959-7174/13/4/009, 2003.

Romeiser, R., and D.R. Thompson, Numerical study on the along-track interferometric radar imaging mechanism of oceanic surface currents, IEEE Trans. on Geosci. and Remote Sensing, 38-II, 446-458, 2000.

Romeiser, R., M. Schwaebisch, J. Schulz-Stellenfleth, D.R. Thompson, R. Siegmund, A. Niedermeier, W. Alpers, and S. Lehner, Study on Concepts for Radar Interferometry from Satellites for Ocean (and Land) Applications (KoRIOLiS), Final Report, 112 pp., University of Hamburg, Germany, available at http://www.ifm.zmaw.de/ romeiser/koriolis.htm, 2002.

Spicer, K. R., J. E. Costa, and G. Placzek, Measuring flood discharge in unstable channels using ground-penetrating radar, Geology, 25, 423 426, 1997.

Teague, C.C., D.E. Barrick, P.M. Lilleboe, and R.T. Cheng, Initial river test of a monostatic RiverSonde streamflow measurement system, Proc. IEEE/OES Seventh Working Conf. on Current Meas. Tech., 46-50, 2003.

Thompson, D.R., and J.R. Jensen, Synthetic Aperture Radar Interferometry Applied to Ship-Generated Internal Waves in the 1989 Loch Linnhe Experiment, J. Geophys. Res., 98 (C6), 10259-10269, 1993. 
Wright J.W., A new model for sea clutter, IEEE Trans. Ant. Prop., AP-16(2), 217-223, 1968.

Yamagucyi, T., and K. Niizato, Flood discharge measurement using radio current meter: Japanese Society of Civil Engineers, v. 497/II-28, 41-50, 1994.

Zebker, H.A. and R.M. Goldstein, Topographic mapping from interferometric synthetic aperture radar observations, J. Geophys. Res., 91 (B5), 4993-4999, 1986. 